HIP-1998-04/TH

TUM-HEP-306/98

DPSU-98-98-1

Janurary, 1998

\title{
Bottom-up approach to $B$-parameter in minimal supersymmetric standard model
}

\author{
Yoshiharu Kawamura ${ }^{a, b}$ 円, Tatsuo Kobayashi ${ }^{c, d}$ 円 \\ and \\ Manabu Watanabe ${ }^{b}$ P \\ ${ }^{a}$ Physik Department, Technische Universität München \\ D-85748 Garching, Germany, \\ ${ }^{b}$ Department of Physics, Shinshu University \\ Matsumoto 390, Japan, \\ ${ }^{c}$ Department of Physics, High Energy Physics Division \\ University of Helsinki \\ and \\ ${ }^{d}$ Helsinki Institute of Physics \\ P.O. Box 9 (Siltavuorenpenger $20 \mathrm{C}$ ) \\ FIN-00014 Helsinki, Finland
}

\begin{abstract}
We study $\mu$ and $B$-parameters in the minimal supersymmetric standard model (MSSM) based on the radiative electroweak symmetry breaking scenario using "bottom-up" approach and show how useful our approach is to select a phenomenologically viable model beyond the MSSM under the assumption that the underlying theory is a string model or a gauge-Yukawa unified gauge model.
\end{abstract}

\footnotetext{
${ }^{1}$ e-mail: ykawamu@gipac.shinshu-u.ac.jp, Humboldt Fellow

${ }^{2}$ e-mail: kobayash@rock.helsinki.fi

${ }^{3}$ e-mail: watanabe@azusa.shinshu-u.ac.jp
} 


\section{Introduction}

Supersymmetric (SUSY) extension of the standard model is an attractive candidate beyond the standard model [1]. Much effort has been devoted to study phenomenological aspects of the minimal supersymmetric standard model (MSSM). One of important issues in the MSSM is what is the origin of physical parameters such as soft SUSY breaking parameters and the $\mu$ parameter. Their patterns and magnitudes are supposed to be related to the SUSY breaking mechanism in some underlying theory. For example, the pattern of soft SUSY breaking parameters depends on the structure of couplings between visible sector and hidden sector, and their magnitudes are of order of the gravitino mass $m_{3 / 2}$ in the hidden sector SUSY breaking scenario of supergravity (SUGRA) [2]. It applies to the effective SUGRA derived from superstring theory.

There is another type of interesting scenario to fix the pattern of physical parameters at some high energy scale. It is coupling reduction theory, whose basic concept is to construct renormalization group ( $R G$ ) invariant relations including those between gauge and Yukawa couplings [3]. Thus, hereafter we call this type of model as gauge-Yukawa unification (GYU) model. Recently it has been applied to reduction of dimensionful couplings, i.e., reduction of soft SUSY breaking parameters [4, 5, 6, 7]. In this framework, soft SUSY breaking parameters are given as functions of a gaugino mass $M_{1 / 2}$ in the form of RG invariants.

In any cases, the $\mu$-parameter is in a special position. The $\mu$-term as well as the $B$-term plays a role in electroweak symmetry breaking, but its magnitude has an arbitariness from theoretical viewpoint, because the $\mu$ parameter can, in principle, have a different origin from that of soft SUSY breaking parameters. On the other hand, the condition that the electroweak symmetry is broken down at the weak scale requires that magnitudes of the $\mu$-parameter and soft SUSY breaking parameters should be around the weak scale. Here we encounter the so-called $\mu$ problem [8]; how is the $\mu$-parameter of $O(1) \mathrm{TeV}$ generated? Several interesting solutions have been proposed $[9,8,10,11,12$. Also, recently the study of $\mu$-term has been carried out from several viewpoints [13, 14, 15, 16]. The exploration of the origin of $\mu$-term is one of important subjects because it can give a key to high energy physics beyond the MSSM. It would be possible to comprehend the high energy physics and the origin of $\mu$-term simultaneously using experimental data in 
future. It would be troublesome to scan a whole range of the parameter space on the MSSM. Hence it is meaningful to investigate which type of $\mu$-term generation mechanism is favorable under some phenomenological requirement in advance. In most cases, the $B$-parameter depends on the mechanism of $\mu$-term generation and there are certain relations of the $B$-parameter with other soft SUSY breaking parameters. .f Hence the study on B-parameter is indispensable to quest the source of $\mu$-parameter and other soft SUSY breaking parameters.

If we specify the underlying theory at high energy scale, $M_{X}$, we can check the reality of $\mu$ and $B$-term generation mechanism comparing two kinds of formulae for $\mu$ and $B$-parameters at $M_{X}$ explained just below. One set of formulae is derived from the phenomenological requirement that the electroweak symmetry is broken radiatively. That is, using the minimization conditions of the Higgs potential, we can write down $\mu$ and $B$-parameters in terms of soft Higgs masses and $\tan \beta=\left\langle h_{2}\right\rangle /\left\langle h_{1}\right\rangle$, where $h_{1}$ and $h_{2}$ are neutral components of Higgs doublets. After the introduction of radiative corrections, $\mu$ and $B$-parameters at $M_{X}$ are written as functions of soft scalar masses $m_{k}, A$-parameters $A_{k l m}$, gaugino masses $M_{a}$ and $\tan \beta$. On the other hand, the formulae of SUSY breaking parameters and $\mu$-parameter are written down in terms of parameters $\left(m_{S},\langle F\rangle, \lambda\right)$ in the underlying theory. Here $m_{S}$ and $\langle F\rangle$ are some mass parameters (e.g., $m_{3 / 2}$ in SUGRA and $M_{1 / 2}$ in GYU-model) and some $F$-term condensations characteristic to the SUSY breaking, respectively. The parameters $\lambda$ are coupling constants related to $\mu$-term generation mechanism. Comparing with these two types of formulae for $\mu$ and $B$-parameters at $M_{X}$, we could find a constraint manifold of solutions in the $\left(m_{S},\langle F\rangle, \tan \beta, \lambda\right)$-space for each of $\mu$ and $B$-term generation mechanisms. As a degree of freedom of $\left(m_{S},\langle F\rangle, \lambda\right)$, in general, is much less than the number of soft SUSY breaking parameters, there are several relations among these soft parameters. If the underlying theory leads to such specific relations, the comparison with the formulae would become serious. In this case, some generation mechanisms might be ruled out in certain regions. This "bottom-up" approach to study $\mu$ and $B$ is more powerful than

* In GYU model, the relation between the B-parameter and other soft SUSY breaking parameters is determined by the use of the coupling reduction procedure independent of the $\mu$-term generation mechanism.

$\dagger$ In [17], qualitative properties of the high-energy parameter space are discussed using bottom-up approach, but the comparison with the prediction of specific models, e.g. string 
the "top-down" approach where one $\mu$-term generation mechanism as well as other soft SUSY breaking parameters is fixed at a high energy scale and then the realization of electroweak symmetry breaking is investigated. Actually the study by the bottom-up approach has been done in Ref. [18] for some parameter regions of superstring models in the small $\tan \beta$ scenario.

In this paper, we develop it and show how useful our approach is to select a phenomenologically viable model beyond the MSSM. We apply our strategy, the bottom-up approach, to the MSSM whose underlying theory is superstring model or GYU-grand unified theory (GUT). The investigation is carried out for several parameter regions in both the small and large $\tan \beta$ scenarios.

This paper is organized as follows. In the next section, we explain our strategy to select a realistic $\mu$ and $B$-term generation mechanism. We devide the parameter space of Yukawa couplings into the small and large $\tan \beta$ scenarios. These two regions show different aspects for the $B$-parameter each other. In section 3, we apply our method to the MSSM derived from a certain type of string model or a generic GYU-GUT. Also comments are given for other cases. Section 4 is devoted to conclusions and discussions.

\section{Bottom-up approach}

In this section, we give an outline of our strategy 18. The neutral components $h_{1}$ and $h_{2}$ of Higgs doublets $H_{1}$ and $H_{2}$ have the following potential [20] 21]:

$$
\begin{aligned}
V\left(h_{1}, h_{2}\right)= & m_{1}^{2} h_{1}^{2}+m_{2}^{2} h_{2}^{2}+\left(\mu B h_{1} h_{2}+\text { h.c. }\right) \\
& +\frac{1}{8}\left(g^{2}+g^{\prime 2}\right)\left(h_{1}^{2}-h_{2}^{2}\right)^{2}, \\
m_{1}^{2} \equiv & m_{H_{1}}^{2}+\mu^{2}, \quad m_{2}^{2} \equiv m_{H_{2}}^{2}+\mu^{2}
\end{aligned}
$$

where the values of all parameters correspond to those at the weak scale. Through our analysis, we use the $Z$ boson mass $M_{Z}=91.2 \mathrm{GeV}$ as the weak scale and we neglect the threshold corrections due to the difference among

model, has not been carried out.

$\ddagger$ In [19], the availability of $B$-term is discussed based on radiative symmetry breaking scenario using string model. 
sparticle masses for simplicity. The mass parameters $m_{H_{1}}^{2}$ and $m_{H_{2}}^{2}$ are soft SUSY breaking masses of Higgs scalars whose hypercharges are $-1 / 2$ and $+1 / 2$, respectively, and $\mu$ is a SUSY Higgs mass parameter. The condition for electroweak symmetry breaking is given by

$$
m_{1}^{2} m_{2}^{2}<|\mu B|^{2}
$$

The bounded from below condition along the $D$-flat direction requires

$$
m_{1}^{2}+m_{2}^{2}>2|\mu B| \text {. }
$$

Further the conditions that minimizing the potential are given by

$$
\begin{aligned}
m_{1}^{2}+m_{2}^{2} & =-\frac{2 \mu B}{\sin 2 \beta}, \\
m_{1}^{2}-m_{2}^{2} & =-\cos 2 \beta\left(M_{Z}^{2}+m_{1}^{2}+m_{2}^{2}\right)
\end{aligned}
$$

where we have used the relation $M_{Z}^{2}=\frac{1}{4}\left(g^{2}+g^{\prime 2}\right) v^{2}$ and $v^{2}=\left\langle h_{2}\right\rangle^{2}+\left\langle h_{1}\right\rangle^{2}$.

By the use of stationary conditions (5) and (6), $\mu$ and $B$-parameters are expressed by

$$
\begin{aligned}
& |\mu|=\frac{1}{\sqrt{2}}\left(\frac{m_{H_{1}}^{2}-m_{H_{2}}^{2}}{-\cos 2 \beta}-m_{H_{1}}^{2}-m_{H_{2}}^{2}-M_{Z}^{2}\right)^{1 / 2}, \\
& |B|=\frac{\sin 2 \beta}{2|\mu|}\left(\frac{m_{H_{1}}^{2}-m_{H_{2}}^{2}}{-\cos 2 \beta}-M_{Z}^{2}\right) .
\end{aligned}
$$

At this stage, $|\mu|$ and $|B|$ are functions of $m_{H_{i}}^{2}$ and $\tan \beta$ at the weak scale. Throughout this paper, we assume that there is no new $\mathrm{CP}$ violation source except that from the Yukawa coupling sector. In this case, we can take $A$ and $B$-parameters real after the gaugino mass parameter is made real and positive by the chiral rotation for the gaugino field. We use this convention with $\mu B<0$ made by a suitable phase rotation for Higgs multiplets.

In order to probe underlying theory, we would like to have $\mu\left(M_{X}\right)$ and $B\left(M_{X}\right)$ as functions of other parameters at $M_{X}$. Through our analysis, we take the gauge coupling unification scale $M_{X}=1.7 \times 10^{16} \mathrm{GeV}$ as an energy scale where boundary conditions are imposed. Considering RG flows [21], we can obtain the $\mu$ and $B$ parameters at $M_{X}$ as follows,

$$
\mu_{ \pm}\left(M_{X}\right)= \pm c_{\mu}|\mu|
$$


and

$$
B_{ \pm}\left(M_{X}\right)=\mp|B|+\Delta B
$$

where $c_{\mu}$ and $\Delta B$ are renormalization factors from $M_{Z}$ to $M_{X}$. Here and hereafter $B_{ \pm}\left(M_{X}\right)$ and $\mu_{ \pm}\left(M_{X}\right)$ denote the $B$ and $\mu$ parameters at $M_{X}$, which are consistent with the radiative electroweak symmetry breaking scenario. The radiative corrections $\Delta B$ depend on $M_{a}^{(0)}$ and $A_{k l m}^{(0)}$ at $M_{X}$ as well as $\tan \beta$, while $c_{\mu}$ depends only on $\tan \beta$. Here and hereafter the suffix $(0)$ of the parameter $a^{(0)}$ denotes a value of the parameter $a$ predicted from underlying theory at $M_{X}$. Further here we have used the fact that Yukawa couplings are given as functions of $\tan \beta$ when the fermion masses are fixed. The top quark mass is given as $m_{t}\left(m_{t}\right)=f_{t}\left(m_{t}\right) v \sin \beta / \sqrt{2}$. The pole mass of top quark $m_{t}^{\text {pole }}$ is related to the running mass by $m_{t}^{\text {pole }}=m_{t}\left(m_{t}\right)[1+$ $\left.4 \alpha_{3}\left(m_{t}\right) /(3 \pi)+O\left(\alpha_{3}^{2}\right)\right]$. We use the values $m_{t}=175 \mathrm{GeV}$ and $m_{\tau}=1.78 \mathrm{GeV}$ as the pole masses of top quark and tau lepton from the current experiments. On the bottom quark mass, we impose the condition $f_{b}=f_{\tau}$ at $M_{X}$ on the Yukawa couplings of bottom quark and tau lepton in the case with large tan $\beta$. Because the experimental value of bottom quark still has some uncertainty and large SUSY corrections can be demanded in the case with large $\tan \beta$ [22, 23].

The soft SUSY breaking scalar masses $m_{k}$ at $M_{Z}$ also receive radiative corrections such that

$$
m_{k}^{2}=m_{k}^{(0) 2}+\sum_{a} \xi_{k}^{a} M_{a}^{(0) 2}+\Delta m_{k}^{2}+S_{k}
$$

where $m_{k}^{(0)}$ 's are soft scalar masses at $M_{X}$, the second term represents a renormalization effect due to gauginos of $U(1)_{Y}, S U(2)_{L}$ and $S U(3)_{C}$ whose masses at $M_{X}$ are given as $M_{a}^{(0)}(a=1,2,3)$, i.e., $\sum_{a} \xi_{H_{1}}^{a}=\sum_{a} \xi_{H_{2}}^{a}=0.52$ for the universal gaugino mass $M_{1 / 2}^{(0)}$. The third term $\Delta m_{k}^{2}$ is a renormalization factor due to Yukawa couplings and this is a function of $m_{k}^{(0)}, A_{k l m}^{(0)}$ and $M_{a}^{(0)}$ as well as $\tan \beta$. The fourth term $S_{k}$ is a tadpole contribution due to scalar fields with hypercharge interaction. Further $m_{k}^{(0)}, A_{k l m}^{(0)}$ and $M_{a}^{(0)}$, in general, are given as functions of parameters $m_{S}$ and $\langle F\rangle$ which appears in the underlying theory. Hence $\mu_{ \pm}\left(M_{X}\right)$ and $B_{ \pm}\left(M_{X}\right)$ are written as functions of $m_{S},\langle F\rangle$ and $\tan \beta$. 
On the other hand, if we specify the underlying theory, we can write down formulae of the $\mu$ and $B$-parameters (they are denoted by $\mu^{(0)}$ and $B^{(0)}$ ) and other soft SUSY breaking parameters at $M_{X}$ using $m_{S},\langle F\rangle, \tan \beta$ and other coupling constants $\lambda$ related to $\mu$-term generation mechanism.

Requiring that $B_{ \pm}\left(M_{X}\right)$ and $\mu_{ \pm}\left(M_{X}\right)$ should agree with $\mu^{(0)}$ and $B^{(0)}$, we can find allowed parameter regions for $\left(m_{S},\langle F\rangle, \tan \beta, \lambda\right)$ leading to successful electroweak symmetry breaking and know which type of $\mu$-term generation mechanism is hopeful. This is the outline of our approach to probe realistic $\mu$ and $B$-parameters. Our approach can be more powerful in the presence of some non-trivial relations among soft SUSY breaking parameters at $M_{X}$ because the number of the free parameters $\left(m_{S},\langle F\rangle\right)$ is reduced. Such a restricted situation appears in the MSSM based on a certain type of string model as well as in a generic GYU-GUT. We will study such cases in the next section.

Next we discuss features of $\mu$ and $B$-parameters in the cases with small $\tan \beta$ and large $\tan \beta$, separately.

(1) Small $\tan \beta$ case $(2 \leq \tan \beta \leq 10)$

In this case, the top Yukawa coupling $f_{t}$ is so strong compared with other Yukawa couplings that the effects of other Yukawa couplings can be neglected. Then we have analytical solutions for renormalization factors $c_{\mu}$ and $\Delta B$ at one-loop level. For example, the solutions in the case with universal gaugino mass at $M_{X}$ are given by $[24]^{\S}$

$$
\begin{aligned}
& c_{\mu} \equiv\left(\frac{\alpha_{2}\left(t_{Z}\right)}{\alpha^{(0)}}\right)^{3 / 2}\left(\frac{\alpha_{1}\left(t_{Z}\right)}{\alpha^{(0)}}\right)^{1 / 22}\left(1+6 \alpha_{t}^{(0)} F\left(t_{Z}\right)\right)^{1 / 4}, \\
& \Delta B \equiv 3 A_{t}^{(0)} \frac{\alpha_{t}^{(0)} F\left(t_{Z}\right)}{1+6 \alpha_{t}^{(0)} F\left(t_{Z}\right)} \\
& +M_{1 / 2}^{(0)}\left\{t_{Z}\left(3 \alpha_{2}\left(t_{Z}\right)+\frac{3}{5} \alpha_{1}\left(t_{Z}\right)\right)-\frac{3 \alpha_{t}^{(0)}\left(t_{Z} F^{\prime}\left(t_{Z}\right)-F\left(t_{Z}\right)\right)}{1+6 \alpha_{t}^{(0)} F\left(t_{Z}\right)}\right\}
\end{aligned}
$$

where

$$
\alpha^{(0)} \equiv \frac{g^{(0) 2}}{4 \pi}, \quad \alpha_{t}^{(0)} \equiv \frac{f_{t}^{(0) 2}}{4 \pi},
$$

\footnotetext{
$\S$ See the second reference in $[17$ for the analytic solutions in the case with non-universal gaugino masses at $M_{X}$.
} 


$$
\begin{aligned}
& F\left(t_{Z}\right) \equiv \int_{0}^{t_{Z}}\left(\frac{\alpha_{3}(t)}{\alpha^{(0)}}\right)^{16 / 9}\left(\frac{\alpha_{2}(t)}{\alpha^{(0)}}\right)^{-3}\left(\frac{\alpha_{1}(t)}{\alpha^{(0)}}\right)^{-13 / 99} d t \\
& t_{Z}=(4 \pi)^{-1} \log \frac{M_{X}^{2}}{M_{Z}^{2}}
\end{aligned}
$$

Here $g^{(0)}$ and $f_{t}^{(0)}$ are the gauge coupling and top Yukawa coupling at $M_{X}$, respectively. Using analytical expressions for $\mu_{ \pm}\left(M_{X}\right), B_{ \pm}\left(M_{X}\right)$ and $m_{H_{i}}^{2}$, $\mu_{ \pm}\left(M_{X}\right)$ and $B_{ \pm}\left(M_{X}\right)$ are written down as functions of $\left(m_{k}^{(0) 2}, M_{a}^{(0)}, A_{t}^{(0)}\right.$, $\tan \beta)$.

(2) Large $\tan \beta$ case $(20 \leq \tan \beta \leq 60)$

We calculate renormalization effects numerically in this case because no analytical solutions are known 7 . Here we discuss the large limit of $\tan \beta$. In this limit, $\mu$ and $B$ parameters are approximately expressed by

$$
\begin{aligned}
& |\mu|^{2} \sim-m_{H_{2}}^{2}-\frac{1}{2} M_{Z}^{2}, \\
& |B| \sim \frac{1}{\tan \beta} \frac{m_{H_{1}}^{2}-m_{H_{2}}^{2}-M_{Z}^{2}}{|\mu|} .
\end{aligned}
$$

From the requirement that $|\mu|^{2}$ and $|B|$ should be positive-definite, we have the following two conditions,

$$
\begin{aligned}
& m_{H_{2}}^{2}<-\frac{1}{2} M_{Z}^{2}, \\
& m_{H_{1}}^{2}>m_{H_{2}}^{2}+M_{Z}^{2} .
\end{aligned}
$$

If the magnitude of $\left(m_{H_{1}}^{2}-m_{H_{2}}^{2}-M_{Z}^{2}\right) /|\mu|$ is of $O\left(m_{S}\right),|B|$ is of $O\left(m_{S} / \tan \beta\right)$, which is negligiblely small compared with its radiative corrections of $O\left(m_{S}\right)$. This statement is justified, in most cases, by the comparison of the values of $\left|B\left(M_{Z}\right)\right| / M_{1 / 2}^{(0)}$ with those of $\Delta B / M_{1 / 2}^{(0)}$ practically. The values of $\left|B\left(M_{Z}\right)\right| / M_{1 / 2}^{(0)}$ are given in Fig. 1 for the universal case with $m_{k}^{(0) 2}=M_{1 / 2}^{(0) 2} / 3$ and $A_{f}^{(0)}=-M_{1 / 2}^{(0)}$. (The implication of these conditions will be discussed later.) The typical order of $\left|B\left(M_{Z}\right)\right| / M_{1 / 2}^{(0)}$ is $O\left(10^{-2}\right)$ for $\tan \beta \geq 30$ and, in particular, it is $O\left(10^{-3}\right)$ for $\tan \beta \geq 50$. Note that the values are suppressed,

I The approximate analytical solutions are obtained in the neighborhood of quasi-fixed points of Yukawa couplings [23]. 
comparing with the order $(O(1 / \tan \beta))$ estimated in the general case, because $m_{H_{1}}^{2}-m_{H_{2}}^{2}$ is proportional to $M_{1 / 2}^{(0) 2}$ with a small coefficient in the universal case. The values approach constants in the limit of large gaugino mass because the dependency of $M_{Z}$ in $\left|B\left(M_{Z}\right)\right| / M_{1 / 2}^{(0)}$ becomes small. The relatively small value of $M_{1 / 2}^{(0)}$ is not allowed from the condition (20). For example, $M_{1 / 2}^{(0)}$ should be bigger than about $180 \mathrm{GeV}$ for $\tan \beta=50$.

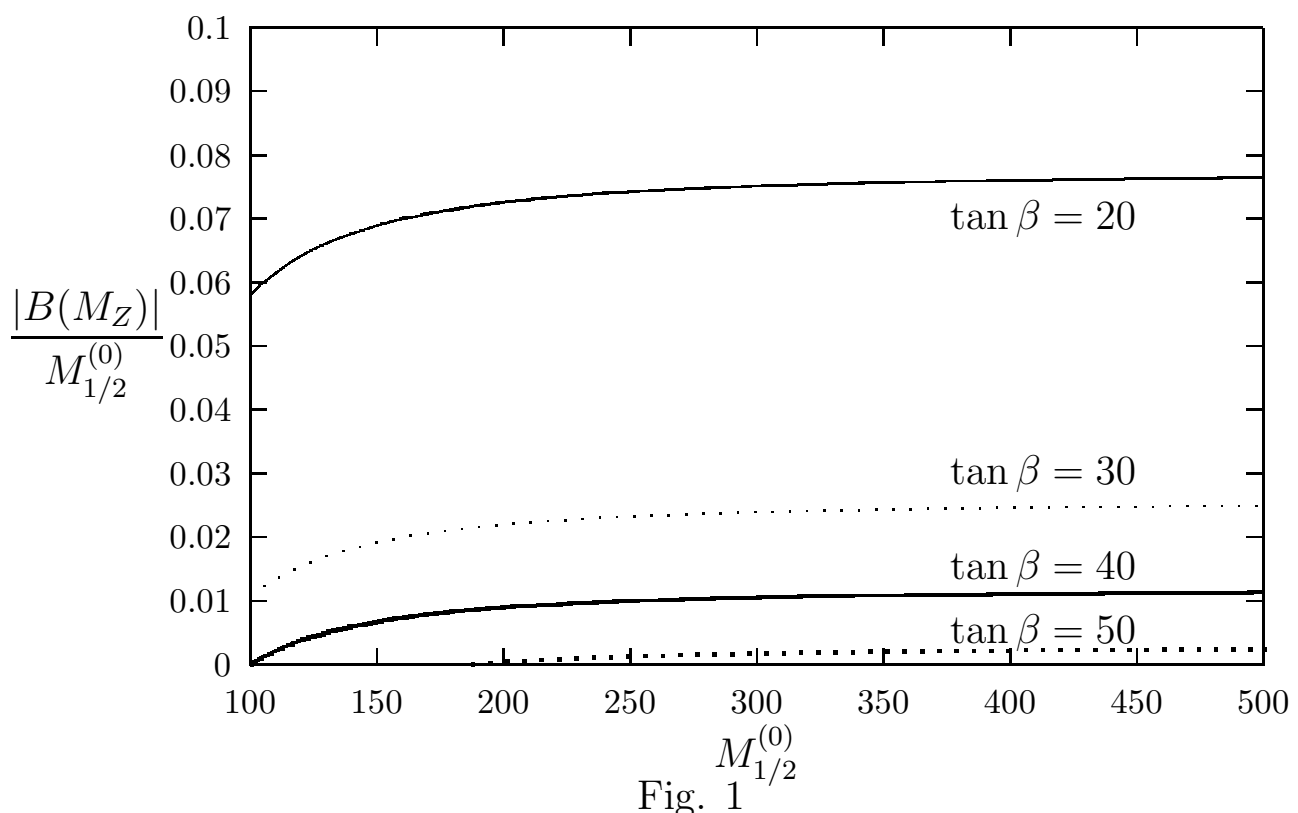

On the other hand, the values of radiative corrections, $\Delta B / M_{1 / 2}^{(0)}$, are shown against $A^{(0)} / M_{1 / 2}^{(0)}$ in Fig. 2, where the solid and dotted lines correspond to the case with $\tan \beta=20$ and 50 , respectively. 


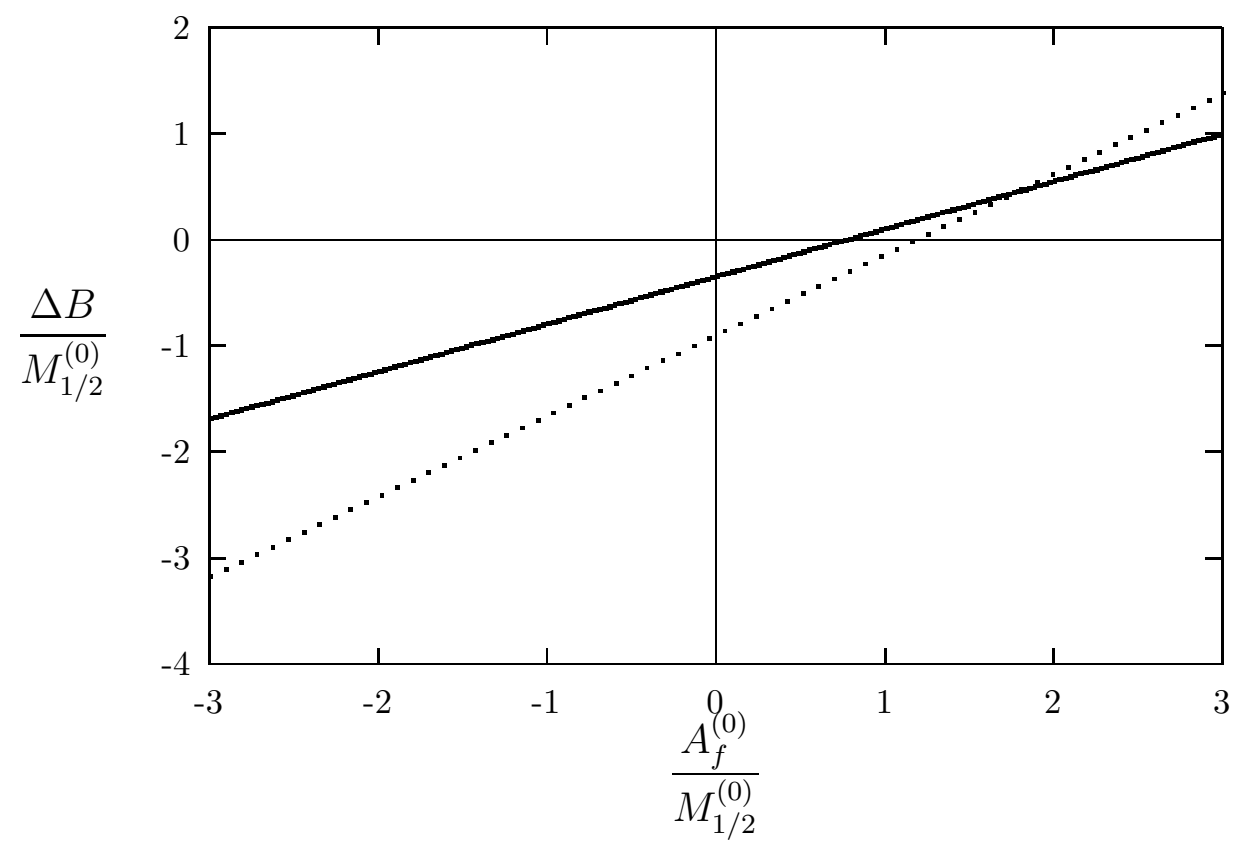

Fig. 2

The typical order of $\Delta B / M_{1 / 2}^{(0)}$ is $O(1)$ except for the case $M_{1 / 2}^{(0)} \sim A_{f}^{(0)}$. Hence we can set $B\left(M_{Z}\right)$ as zero safely for the very large $\tan \beta$ and $M_{1 / 2}^{(0)} \neq A_{f}^{(0)}$. This fact can lead to more powerful prediction in the very large $\tan \beta$ scenario than in the small $\tan \beta$ one because $B_{ \pm}\left(M_{X}\right)$ is strongly dependent of only $M_{a}^{(0)}$ and $A_{k l m}^{(0)}$ in the large $\tan \beta$ scenario, while $m_{k}^{(0)}$ 's are also indispensable to the determination of $B_{ \pm}\left(M_{X}\right)$ in the small $\tan \beta$ scenario. For example, the underlying theory should predict $B^{(0)}$ which satisfies

$$
-1.9 \leq \frac{B^{(0)}}{M_{1 / 2}^{(0)}} \leq-0.7,
$$

for $20 \leq \tan \beta \leq 60$ and $A_{f}^{(0)}=-M_{1 / 2}^{(0)}$, where the value of $B\left(M_{Z}\right) / M_{1 / 2}^{(0)}$ is taken into account for $\tan \beta=20$.

\section{Applications}

As discussed in the previous section, the values $B_{ \pm}\left(M_{X}\right)$ and $\mu_{ \pm}\left(M_{X}\right)$ are functions of $m_{k}^{(0)}, M_{a}^{(0)}$ and $A_{k l m}^{(0)}$ as well as $\tan \beta$. In most cases, some 
underlying thoeries lead to certain specific relations among these parameters, e.g., string-inspired relations, RG-invariant relations or the relations from the no-scale SUGRA model. In this case, our prediction power becomes strong. In this section, we study the origin of $\mu$ and $B$-parameters based on the MSSM with such specific relations from the phenomenological standpoint.

\subsection{Effective MSSM from string theory}

\subsubsection{Formulae from string theory}

At first we discuss a certain type of 4-dimensional string model where SUSY is broken by dilaton and/or moduli $F$-term condensations [25, 26, 27]. In this case, $M_{a}^{(0)}$ and $m_{k}^{(0) 2}$ are given by

$$
\begin{aligned}
& M_{a}^{(0)}=\sqrt{3}\left|m_{3 / 2}\right| \sin \theta e^{-i \alpha_{S}}, \\
& m_{k}^{(0) 2}=\left|m_{3 / 2}\right|^{2}\left(1+n_{k} \cos ^{2} \theta\right)+d_{k}\left|m_{3 / 2}\right|^{2}
\end{aligned}
$$

where $\theta$ is a goldstino angle, $n_{k}$ a modular weight of the corresponding chiral matter field and $d_{k}\left|m_{3 / 2}\right|^{2} D$-term contributions [28]. Here we assume that the vacuum energy vanishes. The universality among gaugino masses originates from the fact that the gauge kinetic functions include only the dilaton field in the same fashion at tree level. In addition, the $A$-parameters are obtained as

$$
A_{k l m}^{(0)}=-\sqrt{3}\left|m_{3 / 2}\right| \sin \theta e^{-i \alpha_{S}}-\left|m_{3 / 2}\right| \cos \theta\left(3+\sum_{k} n_{k}\right) e^{-i \alpha_{T}}
$$

where $\sum_{k} n_{k}$ is the sum of modular weights of matter fields in the corresponding Yukawa couplings $Y_{k l m}$. Here the phases $\alpha_{S}$ and $\alpha_{T}$ stem from phases of $F$-terms of $S$ and $T$. If $Y_{k l m}$ depends on $T$, there appears another

contribution. Here we assume that the top Yukawa coupling is independent of the moduli field. (We will give a brief comment on the case where this assumption is relaxed in 3.3.) Using this assumption and the following relation from $T$-duality of lagrangian

$$
n_{Q_{3}}+n_{t}+n_{H_{2}}=-3
$$

we have the following relations at $M_{X}$ [29],

$$
\begin{aligned}
& A_{t}^{(0)}=-M_{1 / 2}^{(0)}, \\
& m_{\Sigma(t)}^{(0) 2}=m_{\tilde{Q}_{3}}^{(0) 2}+m_{\tilde{t}}^{(0) 2}+m_{H_{2}}^{(0) 2}=M_{1 / 2}^{(0) 2} .
\end{aligned}
$$


In the case with large $\tan \beta$, we assume that the same type of relations as (25), (26) and (27) hold for $A$-parameters and soft SUSY breaking masses related to the bottom and tau Yukawa couplings. In this case, the radiative corrections to $B$ and $m_{k}^{2}$ can be written in terms of $M_{1 / 2}^{(0)}$ as well as $\tan \beta$. On top of that, the initial values of $m_{H_{i}}^{(0) 2}$ can be written in terms of $M_{1 / 2}^{(0)}$ and $\theta$. Thus the values $\mu_{ \pm}\left(M_{X}\right)$ and $B_{ \pm}\left(M_{X}\right)$ can be written down only by the use of $M_{1 / 2}^{(0)}, \tan \beta$ and $\theta$. In the very large $\tan \beta$ scenario, $B_{ \pm}\left(M_{X}\right)$ can be treated as a function of $M_{1 / 2}^{(0)}$ and $\tan \beta$ because the radiative corrections are dominant.

Here we summarize several types of solutions for the $\mu$-problem [27, 30] which we study.

$(\mu-1)$ The $\mu$-parameter $\mu_{Z}^{(0)}$ of $O\left(m_{3 / 2}\right)$ appears after SUSY breaking in the case where a Kähler potential includes a term such as $Z H_{1} H_{2}$ [10]. Hereafter we take $Z=1 /\left(T+T^{*}\right)$ and then $\mu_{Z}^{(0)}$ and $B_{Z}^{(0)}$ are given by

$$
\begin{aligned}
\mu_{Z}^{(0)}= & \left|m_{3 / 2}\right|\left(e^{i \alpha_{3 / 2}}+e^{i \alpha_{T}} \cos \theta\right), \\
B_{Z}^{(0)}= & \frac{\left|m_{3 / 2}\right|}{e^{i \alpha_{3 / 2}}+e^{i \alpha_{T}} \cos \theta}\left\{2-\cos \theta\left(e^{-i\left(\alpha_{T}-\alpha_{3 / 2}\right)}\left(1+n_{H_{1}}+n_{H_{2}}\right)\right.\right. \\
& \left.\left.-e^{i\left(\alpha_{T}-\alpha_{3 / 2}\right)}\right)-\cos ^{2} \theta\left(2+n_{H_{1}}+n_{H_{2}}\right)\right\}
\end{aligned}
$$

where the phase $\alpha_{3 / 2}$ comes from the phase of gravitino mass. The above formula (28) and (29) are obtained from the orbifold models with a multimoduli in the following way. The $Z_{2 n}$ and $Z_{2 n} \times Z_{M}$ orbifold models [31] have $U$-type of moduli fields corresponding to complex structures of orbifolds and a mixing term in the Kähler potential as [32]

$$
\frac{1}{\left(T_{3}+T_{3}^{*}\right)\left(U_{3}+U_{3}^{*}\right)}\left(H_{1} H_{2}+\text { h.c. }\right) \text {. }
$$

In this case, the Higgs fields $H_{1}$ and $H_{2}$ belong to the untwisted sector. The $F$-terms of $S, T_{i}(i=1,2,3)$ and $U_{3}$ are parametrized by $m_{3 / 2}, \theta, \Theta_{i}$ and $\Theta_{3}^{\prime}$ following Refs. [27, 33, 29]. In the case that $\Theta_{3}=1 / \sqrt{3}$ and $\Theta_{3}^{\prime}=0$, the $B$ and $\mu$-parameters reduce to $B_{Z}^{(0)}$ and $\mu_{Z}^{(0)}$, respectively.

$(\mu-2)$ The $\mu$-parameter $\mu_{\lambda}^{(0)}$ of $O\left(m_{3 / 2}\right)$ appears after SUSY breaking in the case where a superpotential $W$ includes a term such as $\lambda \tilde{W} H_{1} H_{2}$ [12]. Here $\tilde{W}$ is a superpotential in the SUSY breaking sector. In this case we 
have

$$
\begin{aligned}
\mu_{\lambda}^{(0)}= & \lambda m_{3 / 2}, \\
B_{\lambda}^{(0)}= & \left|m_{3 / 2}\right|\left\{2 e^{-i \alpha_{3 / 2}}-e^{-i \alpha_{T}} \cos \theta\left(n_{H_{1}}+n_{H_{2}}\right.\right. \\
& \left.\left.-\left\langle T+T^{*}\right\rangle\left\langle\partial^{T} \log \lambda\right\rangle\right)\right\} .
\end{aligned}
$$

( $\mu$-3) The $\mu$-parameter can be generated through some non-perturbative effects such as gaugino condensation [11] and it generally depends on the VEVs of $S$ and $T$. In this case we have

$$
\begin{aligned}
\mu_{\mu}^{(0)}= & \mu_{\mu}(S, T) \\
B_{\mu}^{(0)}= & \left|m_{3 / 2}\right|\left\{-e^{-i \alpha_{3 / 2}}-\sqrt{3} e^{-i \alpha_{S}} \sin \theta\left(1-\left\langle S+S^{*}\right\rangle\left\langle\partial_{S} \log \mu_{\mu}\right\rangle\right)\right. \\
& \left.-e^{-i \alpha_{T}} \cos \theta\left(3+n_{H_{1}}+n_{H_{2}}-\left\langle T+T^{*}\right\rangle\left\langle\partial^{T} \log \mu_{\mu}\right\rangle\right)\right\} .
\end{aligned}
$$

There can be an admixture of several $\mu$-term generation mechanisms and, in this case, $\mu$ and $B$ parameters are given by

$$
\begin{aligned}
\mu_{\mathrm{Mix}}^{(0)} & =\sum_{p} \mu_{p}^{(0)} \\
B_{\mathrm{Mix}}^{(0)} & =\sum_{p} \mu_{p}^{(0)} B_{p}^{(0)} / \sum_{q} \mu_{q}^{(0)}
\end{aligned}
$$

where the indices $p$ and $q$ run over all $\mu$-term generation mechanisms.

We plot $B^{(0)} / M_{1 / 2}^{(0)}$ for $(\mu-1),(\mu-2)$ and $(\mu-3)$ in Fig. 3. Here we restrict ourselves to the case without $\mathrm{CP}$ phases, e.g., $e^{-i \alpha_{S}}= \pm 1$ for $\sin \theta= \pm 1$ and $e^{-i \alpha_{3 / 2}}=e^{-i \alpha_{T}}=1$. (The other simple choice is $e^{-i \alpha_{3 / 2}}=e^{-i \alpha_{T}}=-1$ and the plots in this case are obtained under the reflection for the horizontal axis.) Further we assume that the dependence of $S$ and $T$ is very small in $\mu_{\lambda}^{(0)}$ and $\mu_{\mu}^{(0)}$, i.e., $\partial \lambda / \partial S, \partial \lambda / \partial T, \partial \mu_{\mu}^{(0)} / \partial S, \partial \mu_{\mu}^{(0)} / \partial T \ll 1$. 


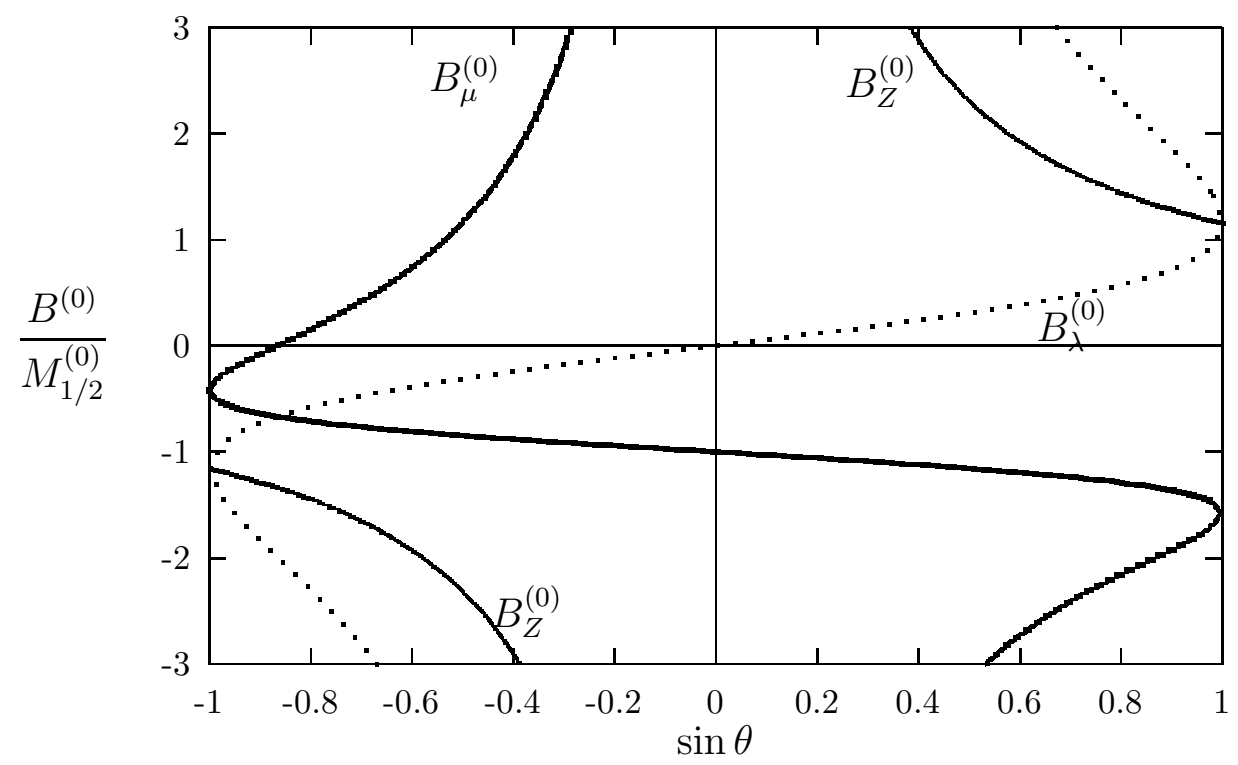

Fig. 3

In general, $B^{(0)} / M_{1 / 2}^{(0)}$ contains a small number of free parameters compared with $\mu^{(0)} / M_{1 / 2}^{(0)}$ and so the analysis of $B^{(0)} / M_{1 / 2}^{(0)}$ can be more predictable.

\subsubsection{Small $\tan \beta$ case}

The parameters $\mu_{ \pm}\left(M_{X}\right)$ and $B_{ \pm}\left(M_{X}\right)$ depend on $m_{k}^{(0) 2}$ in the small $\tan \beta$ scenario and $m_{k}^{(0) 2}$ are functions of $n_{k}, \theta$ and $d_{k}$. First we discuss the simplest case, i.e., the case with the universal soft scalar mass $m^{(0)}$, which satisfies $m^{(0) 2}=M_{1 / 2}^{(0) 2} / 3$. Such universality can be realized in the dilaton-dominant SUSY breaking case or the case where all relevant matter fields have the same modular weight $n_{k}=-1$ in the absence of $D$-term contribution to scalar masses. Fig. 4 and 5 show $B_{ \pm}\left(M_{X}\right) / M_{1 / 2}^{(0)}$ and $\mu_{ \pm}\left(M_{X}\right) / M_{1 / 2}^{(0)}$ against $M_{1 / 2}^{(0)}$ for several values of $\tan \beta=2 \sim 10$. Note that $S_{k}=0$ in the universal case. 


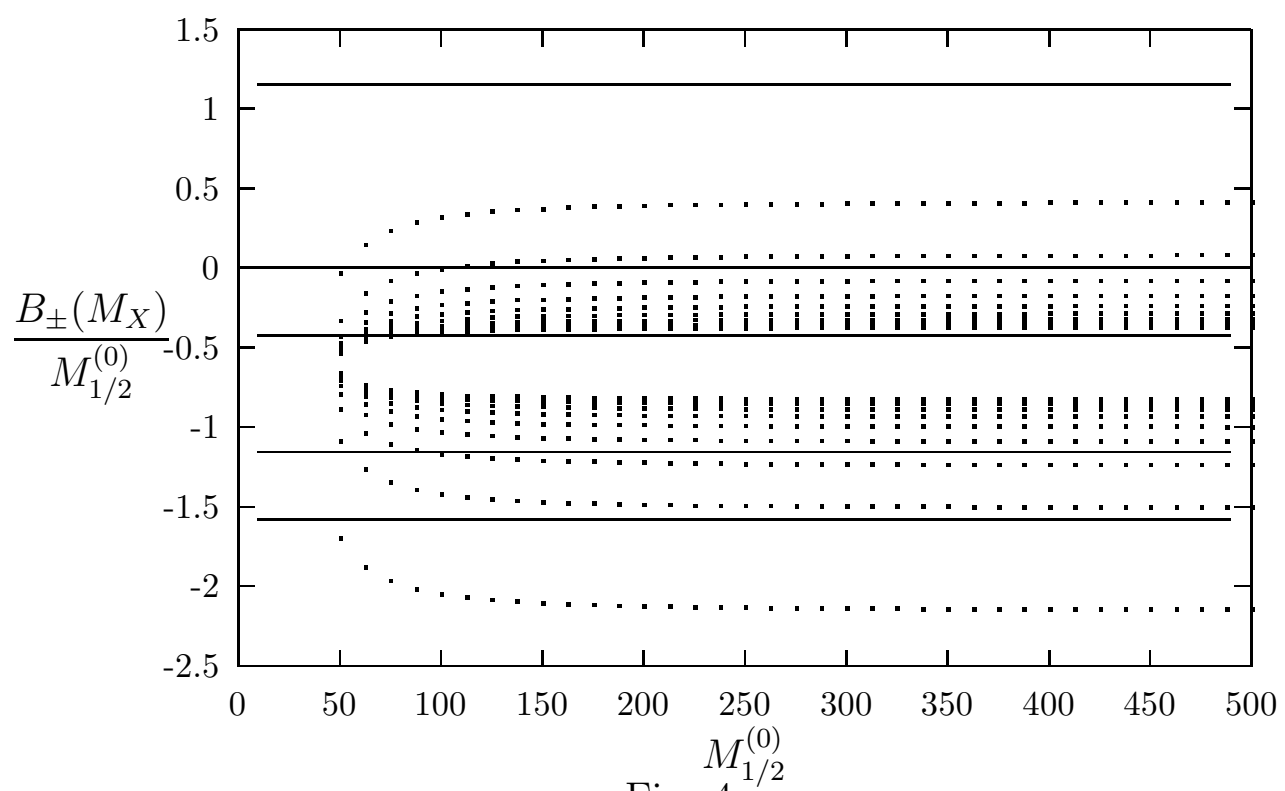

Fig. 4

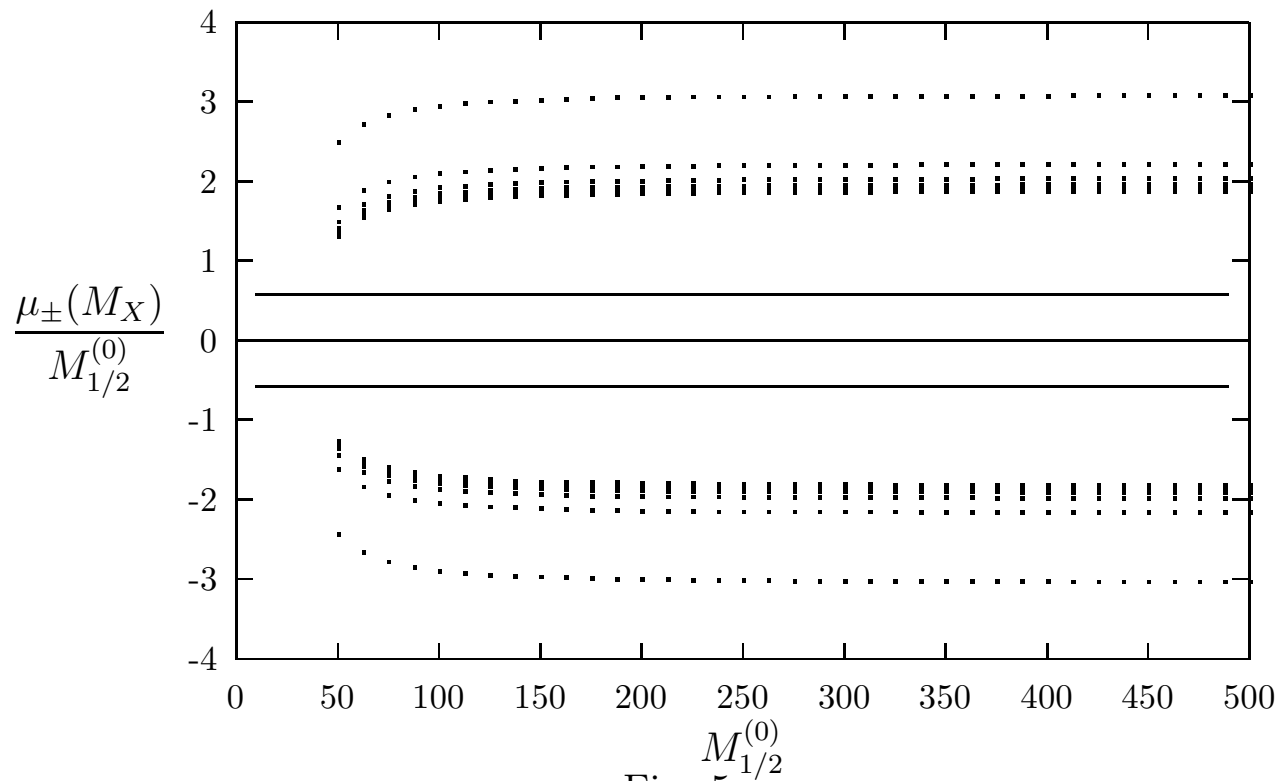

Fig. $5^{1 / 2}$

In both figures, the outside (inside) curves correspond to the case with $\tan \beta=2(10)$. Only in the narrow region where the SUSY threshold is very close to $M_{Z}$, the dependence of $M_{Z} / M_{1 / 2}^{(0)}$ is not weak for $B_{ \pm}\left(M_{X}\right) / M_{1 / 2}^{(0)}$ 
and $\mu_{ \pm}\left(M_{X}\right) / M_{1 / 2}^{(0)}$. However, these values become stable against $M_{1 / 2}^{(0)}$ where $M_{1 / 2}^{(0)}$ is large enough compared with $M_{Z}$.

The ranges of $B_{-}\left(M_{X}\right) / M_{1 / 2}^{(0)}$ and $B_{+}\left(M_{X}\right) / M_{1 / 2}^{(0)}$ are $-0.5 \sim 0.4$ and $-2.2 \sim-0.7$ for $2 \leq \tan \beta \leq 10$. The range of $\left|\mu_{ \pm}\left(M_{X}\right)\right| / M_{1 / 2}^{(0)}$ is $1.4 \sim 3.1$ for $2 \leq \tan \beta \leq 10$. Here we use $M_{1 / 2}^{(0)} \geq 61 \mathrm{GeV}$ as a lower bound of $M_{1 / 2}^{(0)}$, which is derived from the experimental bound on the gluino mass $m_{\tilde{g}} \geq 154$ $\mathrm{GeV}$ [34. The $B$ and $\mu$-parameters can be realistic if the values of $B^{(0)} / M_{1 / 2}^{(0)}$ and $\mu^{(0)} / M_{1 / 2}^{(0)}$ hit the ranges in Fig. 4 and Fig. 5, respectively.

Now let us compare values in Figs. 4 and 5 with values predicted from each $\mu$-term generation mechanism. First we consider the dilaton induced SUSY breaking case $(\sin \theta= \pm 1)$ for concreteness.

The first case $(\mu-1)$ is not realistic since there is no allowed region for $\mu$-parameter as we see from Fig. 4 and $\mu_{Z}^{(0)} / M_{1 / 2}^{(0)}= \pm 1 / \sqrt{3}$. Next we discuss the case with the $D$-term contribution. As discussed in Ref. [28], D-term contribution can survive even in the limit of dilaton dominant SUSY breaking if string model contains an anomalous $U(1)$ symmetry which is cancelled by the Green-Schwarz mechanism [35]. On the other hand, $D$-term contributions related to anomaly-free symmetries vanish at tree level in the limit of dilaton dominant SUSY breaking. Further the tadpole contribution $S_{k}$ can also survive accompanied with $D$-term contributions. It is shown that there are no solutions which satisfy $\mu_{ \pm}\left(M_{X}\right)=\mu_{Z}^{(0)}$ and $B_{ \pm}\left(M_{X}\right)=B_{Z}^{(0)}$ for $2 \leq \tan \beta \leq$ 10 even in the presence of $D$-term contribution for $\mu_{Z}^{(0)} B_{Z}^{(0)}=2 / 3 M_{1 / 2}^{(0) 2}$. On the other hand, we can find solutions after the introduction of $D$-term contribution for $\mu_{Z}^{(0)} B_{Z}^{(0)}=-2 / 3 M_{1 / 2}^{(0) 2}$. Note that the condition $\mu_{Z}^{(0)} B_{Z}^{(0)}=$ $-2 / 3 M_{1 / 2}^{(0) 2}$ is derived by the phase rotation for Higgs multiplets and this case should be also examined based on our convention with $\mu B<0$ at $M_{Z}$.

In the second case $(\mu-2)$, the $\mu$-parameter includes an unknown parameter $\lambda$ and so what we can do is to estimate a favorable value of $\lambda$ using the condition $\mu_{ \pm}\left(M_{X}\right)=\mu_{\lambda}^{(0)}$. There is a solution which satisfies $B_{ \pm}\left(M_{X}\right) / M_{1 / 2}^{(0)}=$ $-2 / \sqrt{3}$ at $\tan \beta \sim 5$. For $B_{ \pm}\left(M_{X}\right) / M_{1 / 2}^{(0)}=2 / \sqrt{3}$, there is a solution at $\tan \beta=1.3$, but it is not realistic since the top Yukawa coupling blows up below $M_{X}$. If we assume the existence of $D$-term contribution, there appears a region which satisfies the condition $B_{ \pm}\left(M_{X}\right) / M_{1 / 2}^{(0)}=2 / \sqrt{3}$ with a positive 
value of $S_{H_{1}}$. I For example, the region with $\tan \beta \sim 2$ and $S_{H_{1}} \sim 2.3 M_{1 / 2}^{(0) 2}$ is allowed. Hence the radiative breaking scenario can be realized in the models with $B_{\lambda}^{(0)} / M_{1 / 2}^{(0)}=-2 / \sqrt{3}$ and/or a contribution of $S_{H_{1}}$ under a suitable value of $\lambda$.

In the third case $(\mu-3), B_{\mu}^{(0)} / M_{1 / 2}^{(0)}$ takes the real values $-(\sqrt{3} \pm 1) / \sqrt{3}$ and $(\sqrt{3} \pm 1) / \sqrt{3}$. We have a solution for $B_{\mu}^{(0)} / M_{1 / 2}^{(0)}=(-\sqrt{3}-1) / \sqrt{3}$ even in the absence of $D$-term and tadpole contributions. The favorable value is $\tan \beta \sim 3$. Similarly, there appears a solution for $B_{\mu}^{(0)} / M_{1 / 2}^{(0)}=(-\sqrt{3}+1) / \sqrt{3}$ at $\tan \beta \sim 10$. At present, though we treat $\mu$ as a free parameter as well as in the second case, we can select a $\mu$-term generation mechanism, when its origin is specified through some non-perturbative effect, by using the allowed region given in Fig. 5 .

We consider the effect of overall moduli $F$-term condensation. The first case $(\mu-1)$ is not realistic without $D$-term contributions. Because the inequality $\left|\mu_{Z}^{(0)} / B_{Z}^{(0)}\right|=|1+\cos \theta| / 2 \leq 1$ derived from Eqs. (28) and (29) is incompatible with the values $\left|\mu_{ \pm}\left(M_{X}\right) / B_{ \pm}\left(M_{X}\right)\right|$ as we see from Figs. 4 and 5 . On the other hand, both of $B_{\lambda}^{(0)}$ and $B_{\mu}^{(0)}$ have solutions of $B_{\lambda(\mu)}^{(0)}=B_{ \pm}\left(M_{X}\right)$ in the wider regions of $\sin \theta$ as well as $\tan \beta$ as we see from Figs. 3 and 4 .

Up to now, we have discussed the case with the universal soft scalar masses except the $D$-term contribution. Similarly we can study cases with non-universal soft scalar masses. Note that radiative corrections do not change from the universal case up to the tadpole and $D$-term contributions as far as the sum rule (27) holds on. Here we consider the case with $n_{H_{1}}=-2$, $n_{H_{2}}=-1$ and $S_{H_{i}}=0$. Fig. 6 shows the values of $B_{ \pm}\left(M_{X}\right) / M_{1 / 2}^{(0)}$ against $\cos \theta$ in the limit $M_{Z} / M_{1 / 2}^{(0)} \rightarrow 0$. Further Fig. 7 shows predicted values of $B^{(0)} / M_{1 / 2}^{(0)}$ from string models with $n_{H_{1}}=-2$ and $n_{H_{2}}=-1$ with a choice $e^{-i \alpha_{3 / 2}}=e^{-i \alpha_{T}}=1$. We can get the allowed values for $\cos \theta$ and $\tan \beta$ comparing with the values in Figs. 6 and 7 for each of $\mu$-term generation mechanisms.

\| The $D$-term contributions to Higgs masses can be absorbed into the tadpole contribution by the redefinition of $S_{H_{i}}$. Here and hereafter we suppose that such a redefinition has been carried out. 


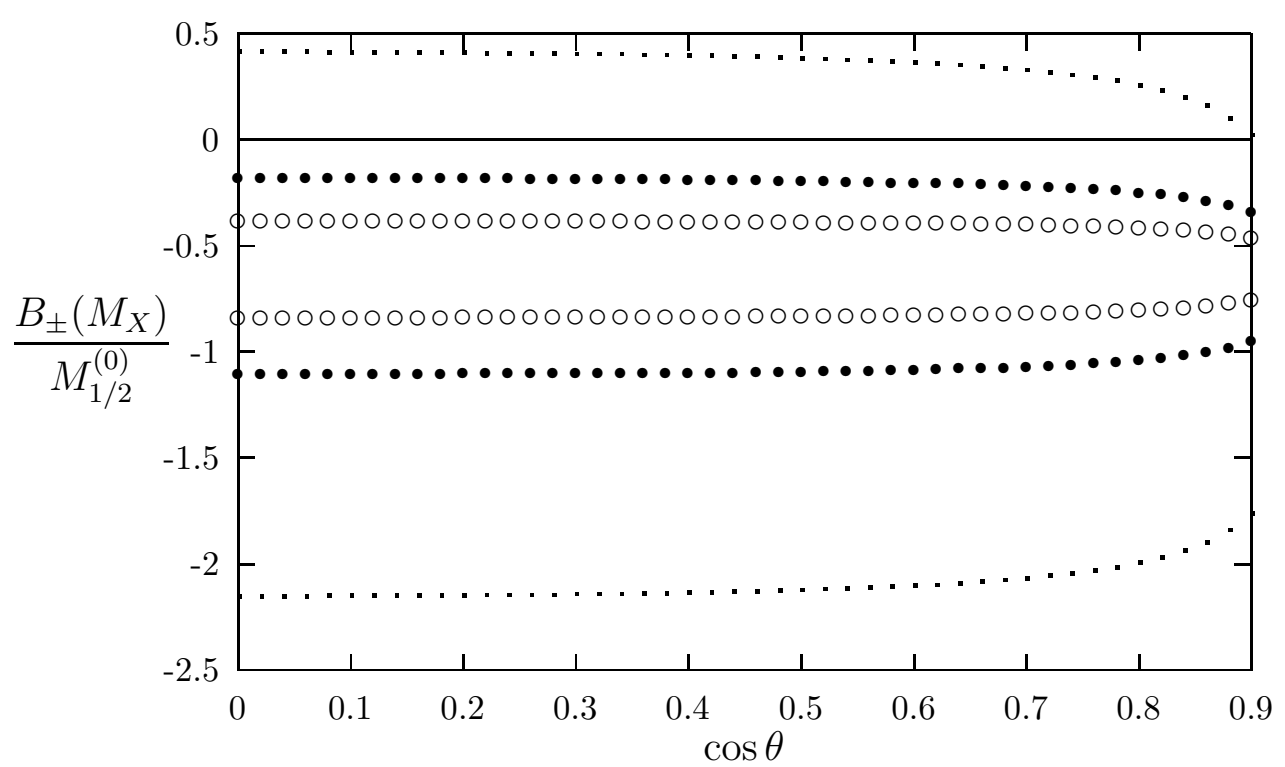

Fig. 6

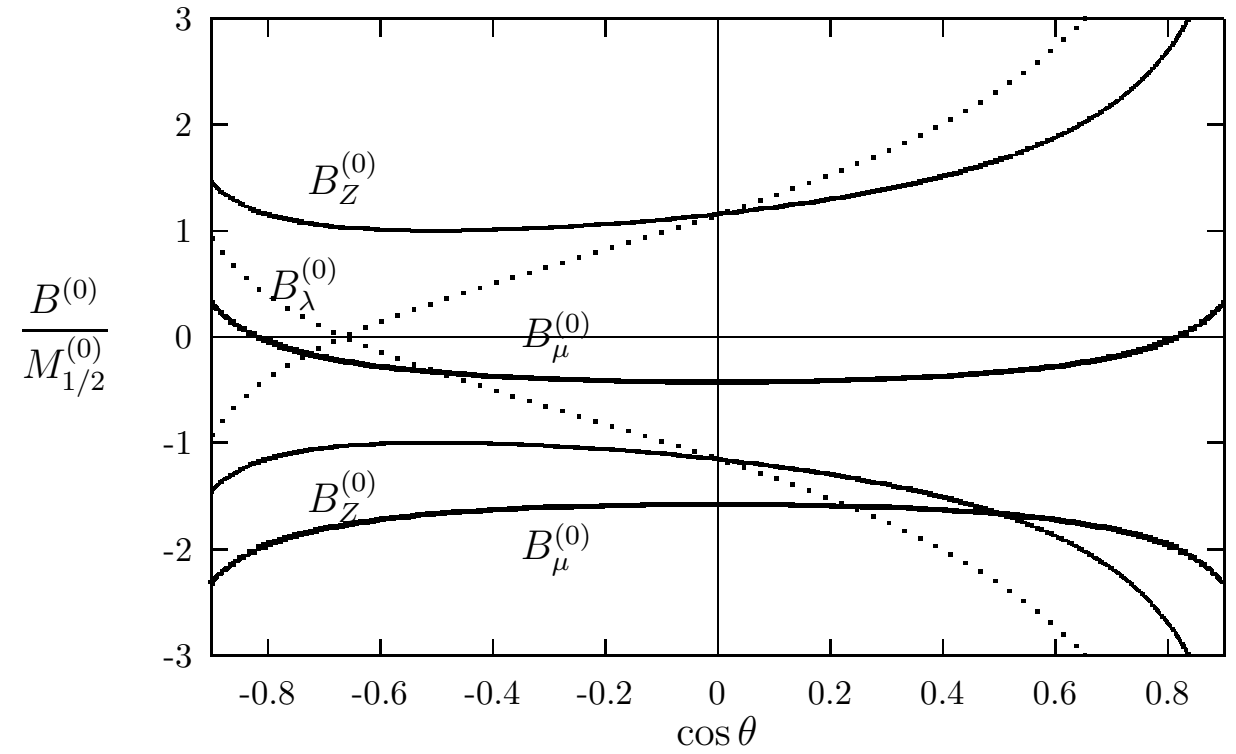

Fig. 7

The difference between the values in the case with $n_{H_{1}}=-1$ and $n_{H_{1}}=-2$ increases as $\cos ^{2} \theta$ increases. Hence the similar conclusion holds for small values of $\cos \theta$ on the reality of the radiative breaking scenario as the case with $n_{H_{1}}=-1$. 


\subsubsection{Large $\tan \beta$ case}

First let us discuss the conditions (19) and (20). If there exists positive sizable $S_{H_{1}}$, the above conditions are fulfilled easily. Even in the universal case, there is allowed region for the large $M_{1 / 2}^{(0) 2}$. Because the radiative correction to the difference $m_{H_{1}}^{2}-m_{H_{2}}^{2}$ is protortional to $M_{1 / 2}^{(0) 2}$ and its coefficient is always positive for $\tan \beta \leq 60$, although such coefficient becomes small for a very large value of $\tan \beta$. For example, we have $0.2 \times M_{1 / 2}^{(0) 2}$ for $\tan \beta=50$ and so it leads to a constraint $M_{1 / 2}^{(0)} \geq 180 \mathrm{GeV}$ as described before.

Second let us analyze the $B$-parameter based on Fig. 8 which shows $\Delta B / M_{1 / 2}^{(0)}$ against $\tan \beta$ in the case with $A_{f}^{(0)}=-M_{1 / 2}^{(0)}$.

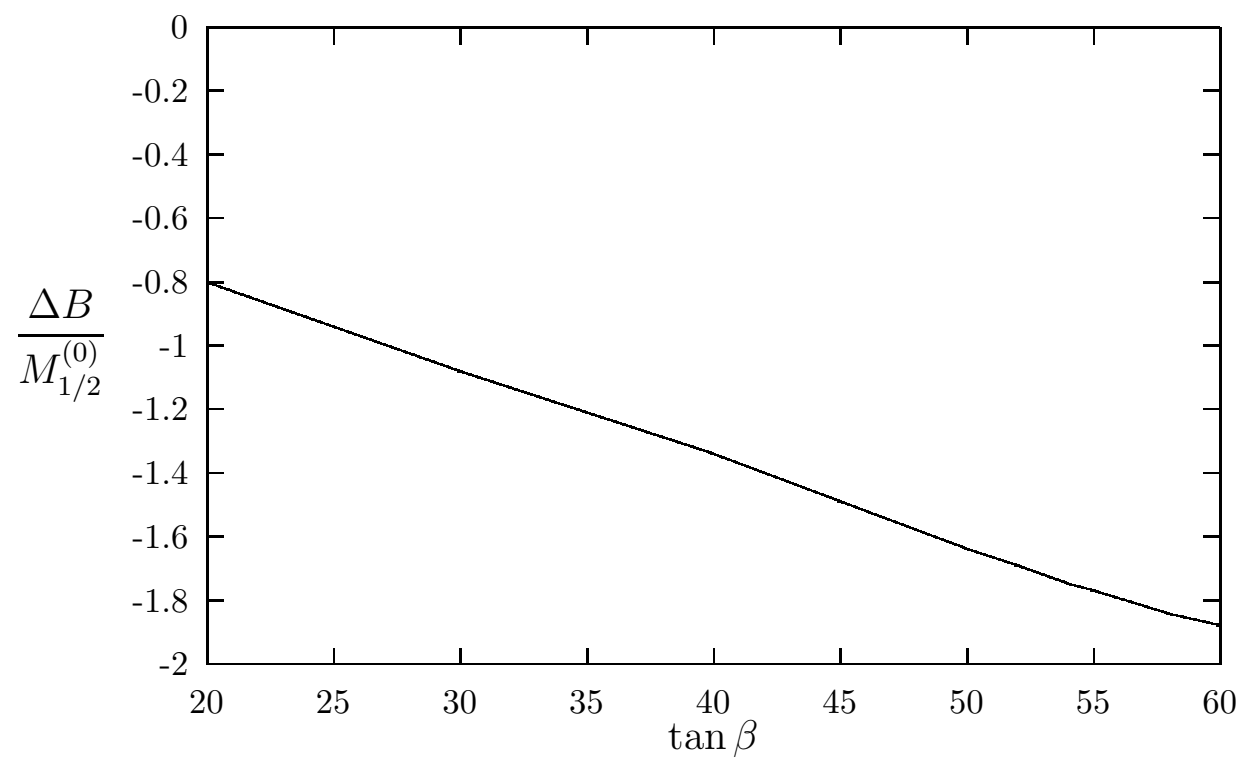

Fig. 8

As mentioned in Eq.(21), the values $B^{(0)} / M_{1 / 2}^{(0)}$ should be within the region $[-1.9,-0.7]$ in the large $\tan \beta$ scenario.

For example, string models with the dilaton dominant SUSY breaking predict $B_{Z}^{(0)} / M_{1 / 2}^{(0)}=B_{\lambda}^{(0)} / M_{1 / 2}^{(0)} \sim \pm 1.15$ and $B_{\mu}^{(0)} / M_{1 / 2}^{(0)} \sim \pm 1.58, \pm 0.42$. Thus, the first and second types of $\mu$-term generation mechanisms cannot realize for $B_{\lambda}^{(0)} / M_{1 / 2}^{(0)} \sim 1.15$ and the third one can realize for $B_{\mu}^{(0)} / M_{1 / 2}^{(0)} \sim$ -1.58. Though there is a solution for $B_{Z}^{(0)} / M_{1 / 2}^{(0)} \sim-1.15$ around $\tan \beta \sim 30$, it is not realistic because we have no solution which satisfies the condition 
$\left|\mu_{ \pm}\left(M_{X}\right)\right| / M_{1 / 2}^{(0)} \sim 0.58$ as we see from Fig. 9. Fig. 9 shows $\left|\mu_{ \pm}\left(M_{X}\right)\right| / M_{1 / 2}^{(0)}$ against $M_{1 / 2}^{(0)}$ for several values of $\tan \beta=20 \sim 60$ where we take the universal case, $m_{k}^{(0) 2}=M_{1 / 2}^{(0) 2} / 3$.

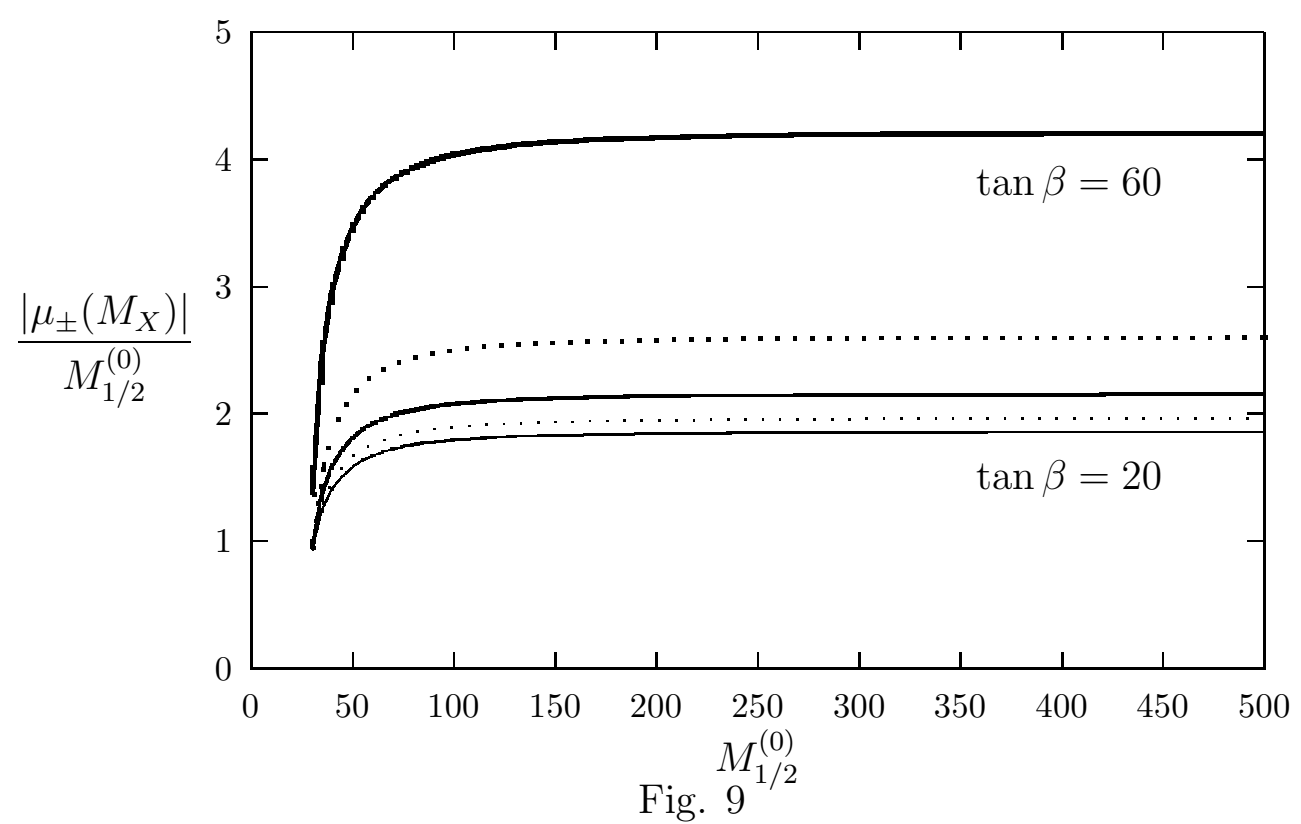

For the second or third ones, we can estimate a favorable value for an unknown parameter in $\mu^{(0)}$ using Fig. 9. The above result holds on except for the case where $B\left(M_{Z}\right)$ is not neglected accidentally.

We study the region of $B$-parameters after incorporating with the effect of overall moduli $F$-term condensation. The first type of $\mu$-term generation mechanism does not realize without $D$-term contributions since $\left|\mu_{Z}^{(0)} / B_{Z}^{(0)}\right|<$ $\left|\mu_{ \pm}\left(M_{X}\right) / B_{ \pm}\left(M_{X}\right)\right|$. For the second one, the region such that $|\sin \theta|>0.9$ is allowed for $B_{\lambda}^{(0)}$. For the third one, Fig. 10 shows $(\tan \beta, \sin \theta)$ which satisfies the condition

$$
\frac{\Delta B}{M_{1 / 2}^{(0)}}=\frac{-1-\sqrt{3} \sin \theta-\cos \theta}{\sqrt{3} \sin \theta}
$$




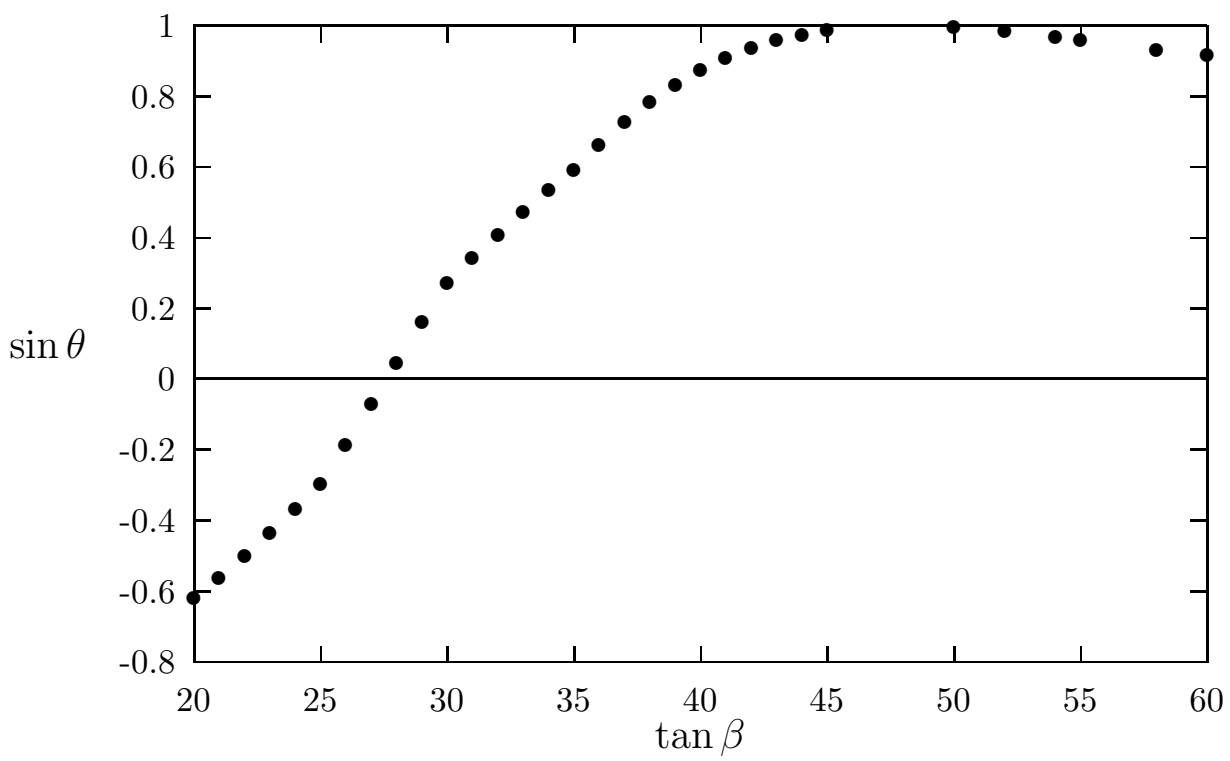

Fig. 10

In addition to the solution shown in Fig. 10, we always have a solution, $\sin \theta \rightarrow 0$ for Eq.(37). In this limit, the parameters $A^{(0)}$ and $M_{1 / 2}^{(0)}$ become very small. Furthermore, the radiative correction $\Delta B$ also becomes small. To obtain precise results in this limit, we need to consider one-loop threshold corrections and $B\left(M_{Z}\right)$.

\subsection{Gauge-Yukawa unification model as theory beyond MSSM}

Recently the coupling reduction technique has been applied to the reduction of soft SUSY breaking parameters [4, 5, 6, 7]. Then it is found that the relations (26) and (27) are RG-invariant. Thus, results in the previous subsection are applicable to GYU-GUTs.

In addition, the following relation [5],

$$
B^{(0)} M_{1 / 2}^{(0)}=-m_{H_{1}}^{(0) 2}-m_{H_{2}}^{(0) 2}
$$

** In Ref. [36], it was found the relations (26) and (27) with the universal soft mass $m_{k}^{(0) 2}=M_{1 / 2}^{(0) 2} / 3$ are two-loop RG invariant in finite models. 
is also RG-invariant and so the following condition should be satisfied,

$$
\frac{B_{ \pm}\left(M_{X}\right)}{M_{1 / 2}^{(0)}}=-h_{H_{1}}-h_{H_{2}}
$$

where $h_{H_{i}}=m_{H_{i}}^{(0) 2} / M_{1 / 2}^{(0) 2}$.

In the universal case, we have $B^{(0)} / M_{1 / 2}^{(0)}=-2 / 3$ [36]. Figs. 4 and 6 show that it is difficult to realize the electroweak breaking scenario in the universal case with the small $\tan \beta$. Similarly it is not realized in the very large $\tan \beta$ scenario as we see from Fig. 2 and 8 . It is known that there is a solution which satisfies $B_{ \pm}\left(M_{X}\right) / M_{1 / 2}^{(0)}=-2 / 3$ for $\tan \beta \sim 18$ [37.

Next we discuss a general case. We can estimate the allowed values for $h_{H_{1}}$ and $h_{H_{2}}$ using Eq. (39) and Figs. 4 and 8 . For example, $h_{H_{1}}+h_{H_{2}}$ should be in $[0.7,1.9]$ for the large $\tan \beta$, e.g. $h_{H_{1}}=h_{H_{2}}=0.8$ for the degenerate case with $\tan \beta=50$. For the small $\tan \beta$, we study the degenerate case, i.e., $h \equiv h_{H_{1}}=h_{H_{2}}$ which includes the universal case as a special one with $h=1 / 3$, and a more general case with non-degenerate soft Higgs masses. Fig. 11 shows the allowed regions for $(\tan \beta, h)$ in the limit $M_{Z} / M_{1 / 2}^{(0)} \rightarrow 0$ for the degenerate case.

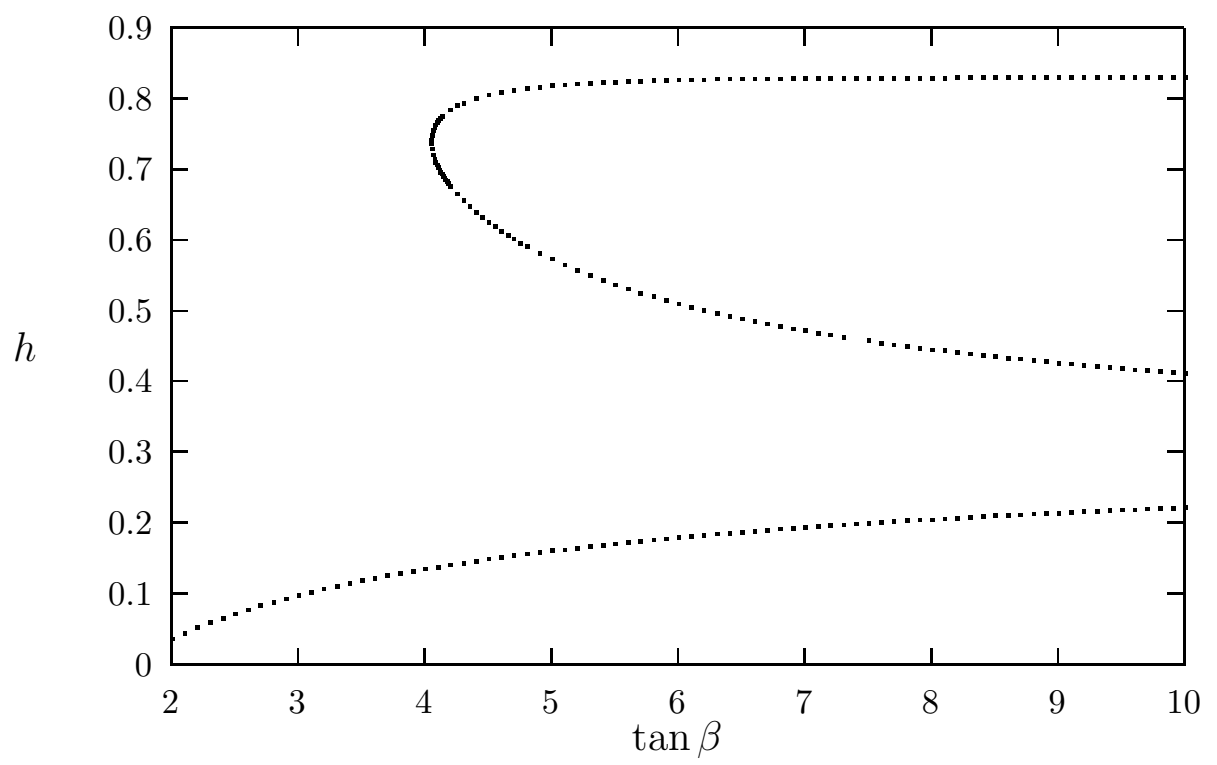

Fig. 11 
For a more general case, solutions satisfying the relation (39) are given in Fig. 12 in the limit $M_{Z} / M_{1 / 2}^{(0)} \rightarrow 0$.

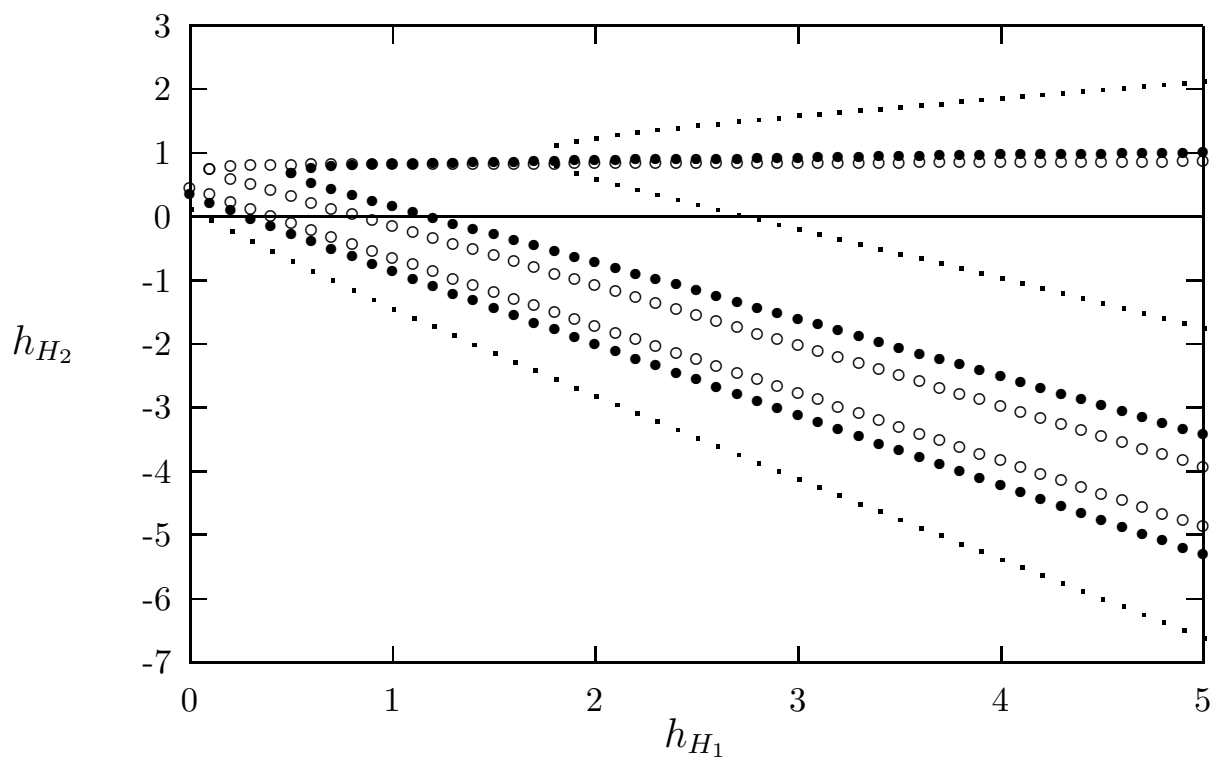

Fig. 12

We can calculate the explicit values of $h_{H_{1}}$ and $h_{H_{2}}$ in the non-finite case if we specify a model of GYU-GUT. It is not obvious whether the values shown in Figs. 11 and 12 can be realized in the explicit model, although in finite models these remain free parameters [6]. Actually there is a restriction for the values of $h_{H_{1}}$ and $h_{H_{2}}$ in a certain situation. In such a case, we can show that it is, in general, difficult to realize radiative electroweak breaking scenario with large $\tan \beta$ in asymptotically free GYU-GUTs under some assumptions as we will discuss in the appendix.

\subsection{Other relations between $M_{1 / 2}^{(0)}$ and $A_{f}^{(0)}$}

Up to now we have discussed the cases with $A_{f}^{(0)}=-M_{1 / 2}^{(0)}$. In this subsection, comments are given for the case with $A_{f}^{(0)} \neq-M_{1 / 2}^{(0)}$. There exist several factors to deviate from the relation $A_{f}^{(0)}=-M_{1 / 2}^{(0)}$, e.g., the existence of the modular weights of matter fields with $\sum n_{k} \neq-3$, the moduli-dependence of the Yukawa couplings in the string model. The Yukawa couplings are 
moduli-independent if the top Yukawa coupling is realized as a renormalizable coupling in the untwisted sector. It is known that the quark doublet $Q_{3}$ and up-type singlet quark $t$ in the third family and the Higgs field $H_{2}$ belong to the untwisted sector in semi-realistic models [38]. (See also Ref. [39].) In other case, the Yukawa couplings include moduli as a volume factor and an exponential factor which are model-dependent.

First we study the violation of the relations (25) and (26) as a trial to make the first or second $\mu$-term generation mechanism in string model realistic from the viewpoint of $B$-parameter in the large $\tan \beta$ scenario. For example, the $A$-parameter is required as $A^{(0)}=2.7 \times M_{1 / 2}^{(0)}$ for $\tan \beta=50$ in order to obtain $\Delta B / M_{1 / 2}^{(0)}=1.15$ as we infer from Fig. 2 . It is impossible to get such large $A$-parameter in the dilaton-dominant SUSY breaking unless the Yukawa couplings have no sizable moduli dependence. Further such a large absolute values of $A$-parameters seems to be unfavorable due to the occurence of dangerous charge and/or color breaking (CCB) 40]. There exists a constraint on the $\left(M_{1 / 2}^{(0)}, A^{(0)}\right)$-plane, i.e., the $\left(\theta, \Sigma n_{k}, \tan \beta\right)$ plane, to satisfy the condition $B_{ \pm}\left(M_{X}\right)=B^{(0)}$. Modular weights of quark and lepton fields are constrained in explicit string models [41, 42]. Thus it is not obvious whether values of $\Sigma n_{k}$ on the constraint plane can be really obtained in explicit string models or not.

Second we discuss the extreme case with $A_{f}^{(0)}=0$. Such an initial condition is realized in the no-scale model [43] and at the same time the no-scale model predicts that soft scalar masses also vanish, $m_{k}^{(0)}=0$. The values of $B_{ \pm}\left(M_{X}\right) / M_{1 / 2}^{(0)}$ in this case, $A_{f}^{(0)}=m_{k}^{(0)}=0$, are plotted in Fig. 13 in the small $\tan \beta$ scenario. 


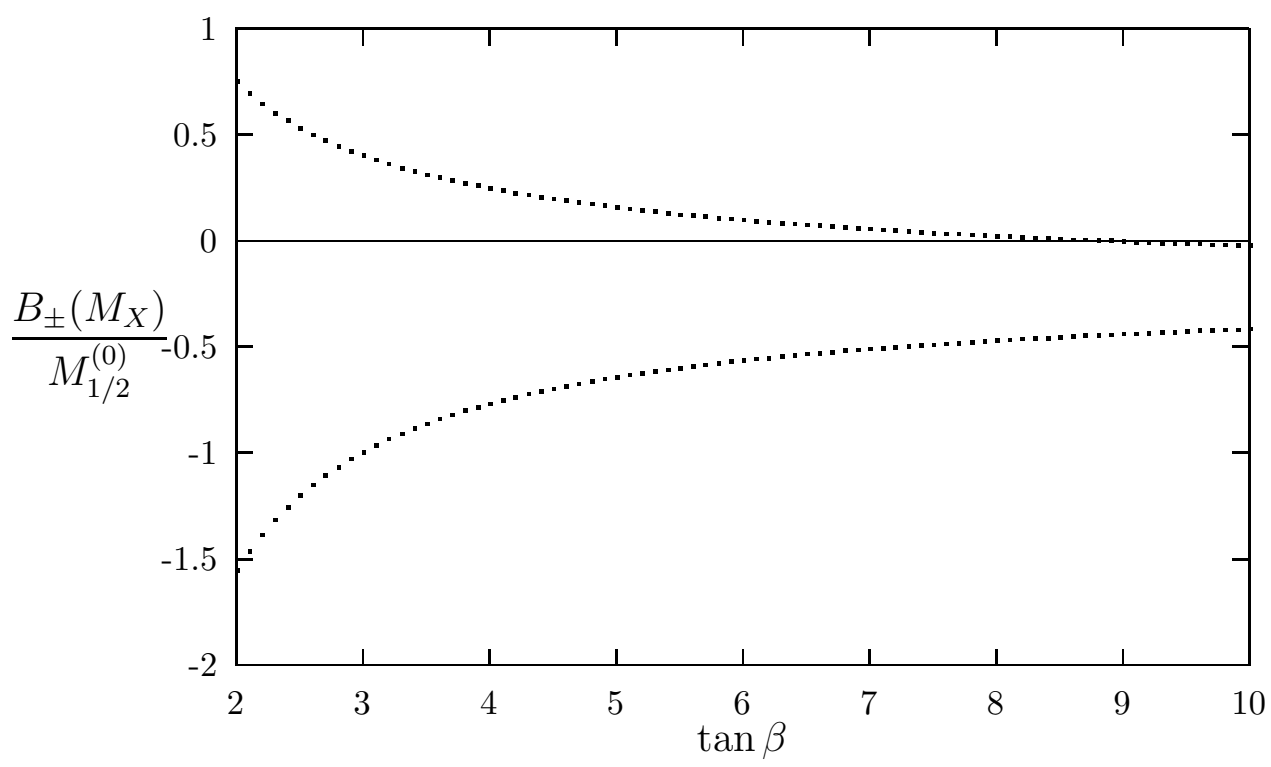

Fig. 13

Here we take a large gaugino mass limit compared with $M_{Z}$. We see that the relation $B^{(0)}=0$ can be realized around $\tan \beta \sim 9$. On the other hand, the condition $B^{(0)}=0$ cannot be realized in the large $\tan \beta$ scenario because $\Delta B / M_{1 / 2}^{(0)}=-0.9 \sim-0.3$ for $\tan \beta=20 \sim 60$ and $A_{f}^{(0)}=0$.

In the case with $0<\left|A^{(0)}\right|<\sqrt{3} M_{1 / 2}^{(0)}$, the value of $\Delta B / M_{1 / 2}^{(0)}$ allows a wider range $[-2,0.5]$ than the universal case, in the large $\tan \beta$ scenario as shown in Fig. 2.

\section{Conclusions and Discussions}

Using the bottom-up approach, we have studied the $\mu$ and $B$ parameters in the framework of the MSSM under the assumption that the underlying theory is a certain type of superstring model or GYU model. These models predict specific relations among soft SUSY breaking parameters, i.e., string-inspired relations and RG invariant relations, which make our predictability strong. Two types of formulae for $\mu$ and $B$-parameters at $M_{X}$ can be written in terms of a few number of independent parameters $\left(m_{S},\langle F\rangle, \tan \beta\right)$. One set is $\left(\mu_{ \pm}\left(M_{X}\right), B_{ \pm}\left(M_{X}\right)\right)$, which is derived from the conditions of the realization of radiative electroweak symmetry breaking. (See Eqs. (9) and (10).) The 
other set is $\left(\mu^{(0)}, B^{(0)}\right)$, which is obtained from the underlying theory. We have examined which type of $\mu$ and $B$-term generation mechanism is hopeful from the requirement that the values from two types of formulae should agree with.

In the MSSM inspired by a certain type of string model, the $\mu$-term generated by the non-renormalizable term such as $\lambda \tilde{W} H_{1} H_{2}$, some nonperturbative effects or both cases, i.e., $(\mu-2),(\mu-3)$ or both cases, is hopeful even in the dilaton dominant SUSY breaking without $D$-term contribution. We have discussed effects of the moduli $F$-term condensation and $D$-term contribution to soft scalar masses. The first mechanism is impossible to realize the radiative scenario without $D$-term contributions even after the introduction of over-all moduli $F$-term contribution. For the second and third ones, we have solutions of $B_{\lambda(\mu)}^{(0)}=B_{ \pm}\left(M_{X}\right)$ in the wider regions of $\sin \theta$ and $\tan \beta$ than the dilaton dominant SUSY breaking case. For $\mu$-parameter, we can select a $\mu$-term generation mechanism, when its origin is specified through some non-perturbative effect, by using the allowed region for $\mu_{ \pm}^{(0)} / M_{1 / 2}^{(0)}$ plotted in Figs. 5 and 9.

In the MSSM based on GYU-GUTs with the reduced $B$-parameter (See Eq.(38)), the small or intermediate $\tan \beta$ scenario is favorable to realize the radiative electroweak symmetry breaking. There is a solution for Eqs. (5) and (6) at $\tan \beta \sim 18$ in the universal case, i.e., $m_{i}^{(0) 2}=M_{1 / 2}^{(0) 2} / 3$ and $A_{f}^{(0)}=$ $-M_{1 / 2}^{(0)}$. We have studied the case with the non-universal masses only for a small $\tan \beta$ region because it is difficult to realize in the large $\tan \beta$ scenario in the framework of asymptotically free GYU-GUTs under some assumptions.

We have also discussed $B$-parameter under the initial condition, i.e., $A_{f}^{(0)}=m_{k}^{(0)}=0$ predicted by the no-scale model. The relation $B^{(0)}=0$ can be realized around $\tan \beta \sim 9$.

Our bottom-up approach to select a realistic $\mu$ and $B$-term generation mechanism is so generic and powerful that we can apply it to the models where the formulae of soft SUSY breaking terms are derived, the case with an improvement of approximation and more complex situations. For example, the improvement by the incorporation of 1-loop effective potential [44, the case with large moduli-dominant threshold corrections for gaugino masses, other assignments of modular weight for matter fields, the case where the coupling constants in $\mu$ and $B$ parameters depend on $S$ and/or $T_{i}$ fields, the modular dominant SUSY breaking case 445 and $\mu$ and $B$-term generation 
mechanism by one-loop effects in SUGRA [15].

\section{Acknowledgments}

This work was partially supported by the European Commission TMR programs ERBFMRX-CT96-0045 and CT96-0090. We would like to thank J. Kubo, H.P. Nilles and M. Olechowski for useful discussions and suggestions.

\section{Appendix}

In this appendix, we show that it is impossible to realize radiative electroweak breaking scenario with large $\tan \beta$ in the framework of asymptotically free GYU-GUTs under some assumptions.

First the formula of soft SUSY breaking scalar masses is given by

$$
m_{k}^{(0) 2}=\frac{2 C_{2}\left(R_{k}\right)-\sum_{f} y_{f} N_{k}^{f}}{-b} M_{1 / 2}^{(0) 2} \equiv h_{k} M_{1 / 2}^{(0) 2}
$$

up to $D$-term contribution due to extra gauge symmetry breaking. Here $C_{2}\left(R_{k}\right)$ is the quadratic Casimir invariants of representation $R_{k}, y_{f}$ the coefficient in the GYU conditions, i.e., $\alpha_{f}^{(0)}=y_{f} \alpha^{(0)}, N_{k}^{f}$ the number of independent diagrams contributed to the wave function renormalization due to the Yukawa coupling $\alpha_{f}^{(0)}$ and $b$ the coefficient of $\beta$ function of the gauge coupling. Note that $y_{f}$ should be positive-definite by definition. We can show the model-independent sum rule, $m_{\Sigma(f)}^{(0) 2}=M_{1 / 2}^{(0) 2}$, by using the following relation derived from the GYU conditions

$$
\sum_{k} \sum_{f} y_{f} N_{k}^{f}=2 \sum_{k} C_{2}\left(R_{k}\right)+b .
$$

Next let us list our basic assumptions in GYU-GUT based on $S U(5)$ or $S O(10)$ gauge group.

1. The theory is asymptotically free. The magnitude of Yukawa coupling related to the bottom quark is comparable to that related to the top quark. 
2. The squarks in the third generation, $\tilde{q}_{3}, \tilde{t}$ and $\tilde{b}$, and Higgs fields $H_{i}$ belong to the following representations in $S U(5)$ and $S O(10)$-GUT, respectively,

$$
\begin{aligned}
& \tilde{q}_{3}, \tilde{t} \in \mathbf{1 0}, \quad \tilde{b} \in \mathbf{5}^{*}, \quad H_{1} \in \mathbf{5}^{*}, \quad H_{2} \in \mathbf{5} \quad \text { under } S U(5), \\
& \tilde{q}_{3}, \tilde{t}, \tilde{b} \in \mathbf{1 6}, \quad H_{1}, H_{2} \in \mathbf{1 0} \quad \text { under } S O(10)
\end{aligned}
$$

3. The multiplets including $\tilde{q}_{3}, \tilde{t}$ and $\tilde{b}$ have no sizable Yukawa couplings other than those among Higgs multiplets including $H_{i}$. However, through sizable Yukawa interactions, the Higgs multiplets including $H_{i}$ can couple to other multiplets, which trigger the breakdown of GUT symmetry, in order that extra Higgs fields acquire heavy masses.

The sum rule, $m_{\Sigma(f)}^{(0) 2}=M_{1 / 2}^{(0) 2}$, is equivalent to the following relations,

$$
\begin{aligned}
& h_{H_{1}}+h_{\tilde{q}_{3}}+h_{\tilde{b}}=1, \\
& h_{H_{2}}+h_{\tilde{q}_{3}}+h_{\tilde{t}}=1 .
\end{aligned}
$$

Let us consider GYU-SU(5)-GUT. The coefficients $h_{k}$ are given by

$$
\begin{aligned}
& h_{\tilde{q} 3}=h_{\tilde{t}}=\frac{1}{-b}\left(\frac{36}{5}-3 y_{t}-2 y_{b}\right), \\
& h_{\tilde{b}}=\frac{1}{-b}\left(\frac{24}{5}-4 y_{b}\right), \\
& h_{H_{1}}=\frac{1}{-b}\left(\frac{24}{5}-4 y_{b}-\sum_{f}^{\prime} y_{f} N_{H_{1}}^{f}\right), \\
& h_{H_{2}}=\frac{1}{-b}\left(\frac{24}{5}-3 y_{t}-\sum_{f}^{\prime} y_{f} N_{H_{2}}^{f}\right)
\end{aligned}
$$

where $\sum^{\prime}$ means the omission of the contribution of the top and bottom Yukawa couplings. The following inequality is derived

$$
h_{H_{1}}+h_{H_{2}}<\frac{7}{13}
$$

from the relations (44) - (49) and $b<0$. Hence we get the inequality $B^{(0)} / M_{1 / 2}^{(0)}>-7 / 13$. It is incompatible to the result $-1.9 \leq B\left(M_{X}\right) / M_{1 / 2}^{(0)} \leq$ -0.7 from the radiative electroweak breaking scenario with large $\tan \beta$. 
In the same way, it is shown that $B^{(0)} / M_{1 / 2}^{(0)}>-2 / 3$ in GYU-SO(10)GUT with the reduced $B$-parameter. The same argument applies to a more generic asymptotically free GUT with the following features.

(1) Every field (the third generation squarks $Q_{3 j}$ and Higgs fields $H_{i}$ ) does not realize as a mixing state but belongs to a single representation. Let us denote them $R_{Q_{3 j}}$ and $R_{H_{i}}$, respectively, bearing the case where some fields belong to the same representation in mind.

(2) The dimensions of $R_{Q_{3 j}}$ equal to or are bigger than those of $R_{H_{i}}$.

(3) Every multiplet including $Q_{3 j}$ has only one sizable Yukawa coupling, which couples to $H_{1}$ and/or $H_{2}$.

The second feature leads to

$$
C_{2}\left(R_{H_{i}}\right) \leq C_{2}\left(R_{Q_{3 j}}\right)
$$

The third feature leads to the following inequality

$$
\sum_{f} y_{f} N_{H_{i}}^{f}>\sum_{f} y_{f} N_{Q_{3 j}}^{f}
$$

Using (51) and (52), we can get the inequality $h_{H_{i}}<h_{Q_{3 j}}$. Further considering the sum rules (44) and (45), we find that both of $h_{H_{i}}$ 's are less than $1 / 3$, which means $B^{(0)} / M_{1 / 2}^{(0)}>-2 / 3$. The presence of $D$-term contribution does not change the above conclusion as far as the quantum numbers of $H_{1}$ for broken diagonal generators take the opposite values of those of $\mathrm{H}_{2}$.

\section{References}

[1] For a review, see H.E. Haber and G.L. Kane, Phys. Rep. 117 (1985) 75.

[2] For a review, see H.P. Nilles, Phys. Rep. 110 (1984) 1.

[3] J. Kubo, M. Mondragón and G. Zoupanos, Nucl. Phys. Proc. Suppl. B56 (1997) 281 and references therein.

[4] J. Kubo, M. Mondragón and G. Zoupanos, Phys. Lett. B389 (1996) 523.

[5] Y. Kawamura, T. Kobayashi and J. Kubo, Phys. Lett. B405 (1997) 64. 
[6] T. Kobayashi, J. Kubo, M. Mondragón and G. Zoupanos, KEK Preprint 97-103, hep-ph/9707425, to be published in Nucl. Phys. B.

[7] Y. Kawamura, T. Kobayashi and J. Kubo, KEK Preprint 97-188, hep$\mathrm{ph} / 9710453$.

[8] J.E. Kim and H.P. Nilles, Phys. Lett. B138 (1984) 150.

[9] H.P. Nilles, M. Srednicki and D. Wyler, Phys. Lett. B120 (1983) 346;

J.M. Frere, D.R.T. Jones and S. Raby, Nucl. Phys. B222 (1983) 11;

J.P. Derendinger and C. Savoy, Nucl. Phys. B237 (1984) 307.

[10] G.F. Giudice and A. Masiero, Phys. Lett. B206 (1988) 480.

[11] J.E. Kim and H.P. Nilles, Phys. Lett. B263 (1991) 79;

E.J. Chun, J.E. Kim and H.P. Nilles, Nucl. Phys. B370 (1992) 105.

[12] J.A. Casas and C. Muñoz, Phys. Lett. B306 (1993) 288.

[13] R. Barbieri, J. Louis and M. Moretti, Phys. Lett. B312 (1993) 451, A. Brignole, L.E. Ibáñez and C. Muñoz, Phys. Lett. B387 (1996) 769.

[14] G.K. Leontaris and N.D. Tracas, Phys. Lett. B394 (1997) 323.

[15] K. Choi, J.S. Lee and C. Muñoz, KAIST-TH 97/13, hep-ph/9709250.

[16] G. Cleaver, M. Cvetič, J.R. Espinosa, L. Everett and P. Langacker, UPR-0750-T,IEM-FT-155/97, hep-ph/9705391.

[17] M. Olechowski and S. Pokorski, Nucl. Phys. B404 (1993) 590;

M. Carena, P. Chankowski, M. Olechowski, S. Pokorski and C.E.M. Wagner, Nucl. Phys. B491 (1997) 103.

[18] Y. Kawamura, T. Kobayashi and M. Watanabe, KEK Preprint 97-11, DPSU-97-5, hep-ph/9705272, to be published in Phys. Lett. B.

[19] S. Khalil, A. Masiero and F. Vassani, Phys. Lett. B375 (1996) 154;

S. Khalil, A. Masiero and Q. Shafi, Phys. Rev. D56 (1997) 5754.

[20] For a review, see L.E. Ibáñez and G.G. Ross, Perspectives in Higgs Physics, ed. G. Kane. 
[21] For radiative breaking scenario of electroweak symmetry, see K. Inoue, A. Kakuto, H. Komatsu and S. Takeshita, Prog. Theor. Phys. 68 (1982) 927; 71 (1984) 413;

L.E. Ibáñez, Phys. Lett. B118 (1982) 73; Nucl. Phys. B218 (1983) 514;

L. Alvarez-Gaumé, J. Polchinski and M. Wise, Nucl. Phys. B221 (1983) 495;

J. Ellis, J.S. Hagelin, D.V. Nanopoulos and K. Tamvakis, Phys. Lett. B125 (1983) 275;

L.E. Ibáñez and C. Lopez, Nucl. Phys. B233 (1984) 511;

L.E. Ibáñez, C. Lopez and C. Muñoz, Nucl. Phys. B256 (1985) 218.

[22] L.J. Hall, R. Rattazzi and U. Sarid, Phys. Rev. D50 (1994) 7048;

R. Hempfling, Phys. Rev. D49 (1994) 6168.

[23] M. Carena, M. Olechowski, S. Pokorski and C.E.M. Wagner, Nucl. Phys. B426 (1994) 269.

[24] K. Hikasa, Minimal Supersymmetry for Collider Physicists, unpublished.

[25] B. de.Carlos, J.A. Casas and C. Muñoz, Phys. Lett. B299 (1993) 234.

[26] V.S. Kaplunovsky and J. Louis, Phys. Lett. B306 (1993) 269.

[27] A. Brignole, L.E. Ibáñez and C. Muñoz, Nucl. Phys. B422 (1994) 125.

[28] Y. Kawamura and T. Kobayashi, Phys. Lett. B375 (1996) 141; Phys. Rev. D56 (1997) 3844;

Y. Kawamura, T. Kobayashi and T. Komatsu, Phys. Lett. B400 (1997) 284.

[29] A. Brignole, L.E. Ibáñez, C. Muñoz and C. Scheich, Z. Phys. C74 (1997) 157.

[30] For review on $\mu$-problem, see, e.g. C. Muñoz, FTUAM 95/20.

[31] L. Dixon, J. Harvey, C. Vafa and E. Witten, Nucl. Phys. B261 (1985) 651 ; B274 (1986) 285;

L.E. Ibáñez, J.E. Kim, H.P. Nilles and F. Quevedo, Phys. Lett. B191 (1987) 282; 
A. Font, L.E. Ibáñez and F. Quevedo, Phys. Lett. B217 (1989) 272;

Y. Katsuki, Y. Kawamura, T. Kobayashi, N. Ohtsubo, Y. Ono and K. Tanioka, Nucl. Phys. B341 (1990) 611;

T. Kobayashi and N. Ohtsubo, Phys. Lett. B262 (1991) 425; Int. J. Mod. Phys. A9 (1994) 87.

[32] G. Lopes-Cardoso, D. Lüst and T. Mohaupt, Nucl. Phys. B432 (1994) 68 ;

I. Antoniadis, E. Gava, K.S. Narain and T.R. Taylor, Nucl. Phys. B432 (1994) 187.

[33] T. Kobayashi, D. Suematsu, K. Yamada and Y. Yamagishi, Phys. Lett. B348 (1995) 402.

[34] Particle Data Group, R.M. Barnett et al., Phys. Rev. D54 (1996) 1.

[35] M.B. Green and J.H. Schwarz, Phys. Lett. B149 (1984) 117;

L.E. Ibáñez, Phys. Lett. B303 (1993) 55.

[36] I. Jack and D.R.T. Jones, Phys. Lett. B333 (1994) 372.

[37] I. Jack, D.R.T. Jones and K.L. Roberts, Nucl. Phys. B455 (1995) 83.

[38] J.A. Casas and C. Muñoz, Phys. Lett. B209 (1988) 214; B214 (1988) 63 ;

A. Font, L.E. Ibáñez, F. Quevedo and A. Sierra, Nucl. Phys. B331 (1990) 421.

[39] K.R. Dienes, Phys. Rep. 289 (1997) 447 and references therein.

[40] J.M. Frere, D.R.T. Jones and S. Raby, Nucl. Phys. B222 (1983) 11;

L. Alvarez-Gaumé, J. Polchinski and M. Wise, Nucl. Phys. B221 (1983) 495;

J.P. Derendinger and C.A. Savoy, Nucl. Phys. B237 (1984) 307;

C. Kounnas, A.B. Lahanas, D.V. Nanopoulos and M. Quirós, Nucl. Phys. B236 (1984) 438.

[41] L.E. Ibáñez and D. Lüst, Nucl. Phys. B382 (1992) 305.

[42] H. Kawabe, T. Kobayashi and N. Ohtsubo, Nucl. Phys. B434 (1995) 210. 
[43] For a review, see A.B. Lahanas and D.V. Nanopoulos, Phys. Rep. 145 (1987) 1.

[44] G. Gamberini, G. Ridolfi and F. Zwirner, Nucl. Phys. B331 (1990) 187.

[45] Y. Kawamura, S. Khalil and T. Kobayashi, Nucl. Phys. B502 (1997) 37. 


\section{Figure Captions}

Figure 1 Values of $\left|B\left(M_{Z}\right)\right| / M_{1 / 2}^{(0)}$ against $M_{1 / 2}^{(0)}$ in the universal case with $m_{i}^{(0) 2}=$ $M_{1 / 2}^{(0) 2} / 3$ and $A_{f}^{(0)}=-M_{1 / 2}^{(0)}$.

Figure 2 Values of $\Delta B / M_{1 / 2}^{(0)}$ against $A_{f}^{(0)} / M_{1 / 2}^{(0)}$ in the case with non-universal parameters. The solid and dotted curves correspond to the cases with $\tan \beta=20$ and 60 , respectively.

Figure 3 Values of $B^{(0)} / M_{1 / 2}^{(0)}$ against $\sin \theta$ for $\mu$-term generation mechanisms $(\mu$ $1),(\mu-2)$ and $(\mu-3)$ with a choice $e^{-i \alpha_{3 / 2}}=e^{-i \alpha_{T}}=1$, i.e., $B_{Z}^{(0)} / M_{1 / 2}^{(0)}=$ $2 /(\sqrt{3} \sin \theta), B_{\lambda}^{(0)} / M_{1 / 2}^{(0)}=(2+2 \cos \theta) /(\sqrt{3} \sin \theta)$ and $B_{\mu}^{(0)} / M_{1 / 2}^{(0)}=$ $-(1+\sqrt{3} \sin \theta+\cos \theta) /(\sqrt{3} \sin \theta)$.

Figure 4 Values of $B_{ \pm}\left(M_{X}\right) / M_{1 / 2}^{(0)}$ against $M_{1 / 2}^{(0)}$ in the universal case with $m_{i}^{(0) 2}=$ $M_{1 / 2}^{(0) 2} / 3$ and $A_{t}^{(0)}=-M_{1 / 2}^{(0)}$. The solid lines represent the values, $B^{(0)} / M_{1 / 2}^{(0)}= \pm 2 / \sqrt{3}$ and $(-1 \mp \sqrt{3}) / \sqrt{3}$, predicted from $(\mu-1)-(\mu-$ 3 ) in the dilaton dominant SUSY breaking case. (The lines represent $B^{(0)} / M_{1 / 2}^{(0)}=(1 \pm \sqrt{3}) / \sqrt{3}$ are omitted. $)$

Figure 5 Values of $\mu_{ \pm}\left(M_{X}\right) / M_{1 / 2}^{(0)}$ against $M_{1 / 2}^{(0)}$ in the universal case with $m_{i}^{(0) 2}=$ $M_{1 / 2}^{(0) 2} / 3$ and $A_{t}^{(0)}=-M_{1 / 2}^{(0)}$. The solid line represents the value, $\mu^{(0)} / M_{1 / 2}^{(0)}$ $= \pm 1 / \sqrt{3}$, predicted from $(\mu-1)$.

Figure 6 Values of $B_{ \pm}\left(M_{X}\right) / M_{1 / 2}^{(0)}$ against $\cos \theta$ in the case with $n_{H_{1}}=-2$ and $n_{H_{2}}=-1$ in the limit $M_{Z} / M_{1 / 2}^{(0)} \rightarrow 0$. The dotted curves correspond to the case for $\tan \beta=2$, while curves with closed (open) circles correspond to the case for $\tan \beta=5$ (10).

Figure 7 Values of $B^{(0)} / M_{1 / 2}^{(0)}$ against $\cos \theta$ in the case with $n_{H_{1}}=-2$ and $n_{H_{2}}=$ -1 for $\mu$-term generation mechanisms $(\mu-1),(\mu-2)$ and $(\mu-3)$ with a choice $e^{-i \alpha_{3 / 2}}=e^{-i \alpha_{T}}=1$.

Figure 8 Values of radiative corrections, $\Delta B / M_{1 / 2}^{(0)}$, against $M_{1 / 2}^{(0)}$ in the case with $A_{f}^{(0)}=-M_{1 / 2}^{(0)}$. 
Figure 9 Values of $\left|\mu_{ \pm}\left(M_{X}\right)\right| / M_{1 / 2}^{(0)}$ against $M_{1 / 2}^{(0)}$ for several values of $\tan \beta=$ $20,30,40,50$ and 60 where we take the universal case.

Figure 10 Values $(\tan \beta, \sin \theta)$ which satisfy the condition $\Delta B=B_{\mu}^{(0)}$.

Figure 11 Values $(\tan \beta, h)$ which satisfy the condition $B_{ \pm}\left(M_{X}\right) / M_{1 / 2}^{(0)}=-2 h$ in the limit $M_{Z} / M_{1 / 2}^{(0)} \rightarrow 0$.

Figure 12 Values $\left(h_{H_{1}}, h_{H_{2}}\right)$ which satisfy the condition $B_{ \pm}\left(M_{X}\right) / M_{1 / 2}^{(0)}=-h_{H_{1}}-$ $h_{H_{2}}$ in the limit $M_{Z} / M_{1 / 2}^{(0)} \rightarrow 0$. The dotted curves correspond to the case with $\tan \beta=2$, while curves with closed (open) circles correspond to the case with $\tan \beta=5$ (10).

Figure 13 Values of $B_{ \pm}\left(M_{X}\right) / M_{1 / 2}^{(0)}$ in the case with $A_{f}^{(0)}=m_{k}^{(0)}=0$ and in the $\operatorname{limit} M_{Z} / M_{1 / 2}^{(0)} \rightarrow 0$. 